\title{
RESONANT ROBIN PROBLEMS DRIVEN BY THE $p$-LAPLACIAN PLUS AN INDEFINITE POTENTIAL
}

\author{
Nikolaos S. Papageorgiou, Vicenţiu D. Rădulescu and Dušan D. Repovš
}

\author{
National Technical University, Department of Mathematics \\ Zografou Campus, Athens 15780, Greece; npapg@math.ntua.gr \\ AGH University of Science and Technology, Faculty of Applied Mathematics \\ al. Mickiewicza 30, 30-059 Krakow, Poland \\ and University of Craiova, Department of Mathematics \\ 200585 Craiova, Romania; vicentiu.radulescu@imar.ro \\ University of Ljubljana, Faculty of Education and Faculty of Mathematics and Physics \\ SI-1000 Ljubljana, Slovenia; dusan.repovs@guest.arnes.si
}

\begin{abstract}
We consider a nonlinear Robin problems driven by the $p$-Laplacian plus an indefinite potential. The reaction is resonant with respect to a variational eigenvalue. For the principal eigenvalue we assume strong resonance. Using variational tools and critical groups we prove existence and multiplicity theorems.
\end{abstract}

\section{Introduction}

Let $\Omega \subseteq \mathbf{R}^{N}$ be a bounded domain with a $C^{2}$-boundary $\partial \Omega$. In this paper, we study the following nonlinear Robin problem

$$
\begin{cases}-\Delta_{p} u(z)+\xi(z)|u(z)|^{p-2} u(z)=f(z, u(z)) & \text { in } \Omega \\ \frac{\partial u}{\partial n_{p}}+\beta(z)|u|^{p-2} u=0 & \text { on } \partial \Omega .\end{cases}
$$

In this problem, $\Delta_{p}$ denotes the $p$-Laplace differential operator defined by

$$
\Delta_{p} u=\operatorname{div}\left(|D u|^{p-2} D u\right) \text { for all } u \in W^{1, p}(\Omega) .
$$

The potential function $\xi(\cdot) \in L^{\infty}(\Omega)$ is indefinite (that is, sign changing) and the reaction term $f(z, x)$ is a Carathéodory function (that is, for all $x \in \mathbf{R}, z \mapsto f(z, x)$ is measurable and for almost all $z \in \Omega, x \mapsto f(z, x)$ is continuous). In the boundary condition, $\frac{\partial u}{\partial n_{p}}$ denotes the generalized normal derivative corresponding to the $p$ Laplace differential operator and is defined by extension of the map

$$
u \mapsto \frac{\partial u}{\partial n_{p}}=|D u|^{p-2}(D u, n)_{\mathbf{R}^{N}} \text { for all } u \in C^{1}(\bar{\Omega}),
$$

with $n(\cdot)$ being the outward unit normal on $\partial \Omega$. The boundary coefficient term is $\beta \in C^{0, \alpha}(\partial \Omega)$ with $\alpha \in(0,1)$ and $\beta(z) \geqslant 0$ for all $z \in \partial \Omega$. The case $\beta \equiv 0$ corresponds to the Neumann problem.

Our aim here is to investigate the existence and multiplicity of nontrivial smooth solutions for problem (1) when resonance occurs, namely when the function $\frac{f(z, x)}{|x|^{p-2} x}$ asymptotically, as $x \rightarrow \pm \infty$, hits a variational eigenvalue of $-\Delta_{p}+\beta(z) I$ with Robin boundary condition (here, $I$ denotes the identity operator). In the case of resonance

https://doi.org/10.5186/aasfm.2018.4331

2010 Mathematics Subject Classification: Primary 35J20, 35J60, 58E05.

Key words: $p$-Laplacian, indefinite potential, resonance, strong resonance, variational eigenvalue, nonlinear regularity, critical groups, Robin boundary condition. 
with respect to the principal (first) eigenvalue, we consider problems with "strong" resonance, namely we have

$$
f(z, x)=\hat{\lambda}_{1}|x|^{p-2} x+g(z, x)
$$

with $\hat{\lambda}_{1}$ being the first eigenvalue and $g(z, x)$ a Carathéodory perturbation satisfying

$$
\lim _{x \rightarrow \pm \infty} g(z, x)=0 \quad \text { and } \quad \lim _{x \rightarrow \pm \infty} \int_{0}^{x} g(z, s) d s \in \mathbf{R} .
$$

It is well-known that this class of resonant problems presents a special interest since the energy functional of the problem exhibits a partial lack of compactness.

Recently, Neumann problems (that is, $\beta \equiv 0$ ) with an indefinite potential have been investigated by Mugnai and Papageorgiou [16] and Papageorgiou and Rădulescu $[17,19]$. Resonant problems have been considered by Mugnai and Papageorgiou [16], who have been dealt with problems resonant at the first eigenvalue but did not cover the strongly resonant case. Strongly resonant semilinear Dirichlet problems with zero potential have been studied by Landesman and Lazer [12] (they coined the term "strong resonance"), Thews [22], Bartolo, Benci and Fortunato [2], Ward [24] (existence of solutions) and Goncalves and Miyagaki [10] (multiplicity of solutions).

Our approach is based on variational tools coming from the critical point theory and on Morse theory (critical groups). In the next section, for the convenience of the reader, we recall some basic definitions and facts from both theories which we will need in the sequel and we fix our notation.

\section{Mathematical background-preliminary results}

Let $X$ be a Banach space. By $X^{*}$ we denote the topological dual of $X$ and by $\langle\cdot, \cdot\rangle$ we denote the duality brackets for the pair $\left(X^{*}, X\right)$. Given $\varphi \in C^{1}(X, \mathbf{R})$, we say that $\varphi$ satisfies the "Cerami condition at the level $c \in \mathbf{R}$ " (the " $C_{c}$-condition", for short), if the following property holds:

"Every sequence $\left\{u_{n}\right\}_{n \geqslant 1} \subset X$ such that

$$
\varphi\left(u_{n}\right) \rightarrow c \text { and }\left(1+\left\|u_{n}\right\|\right) \varphi^{\prime}\left(u_{n}\right) \rightarrow 0 \text { in } X^{*} \text { as } n \rightarrow \infty,
$$

admits a strongly convergent subsequence".

Proposition 1. Assume that $\varphi \in C^{1}(X, \mathbf{R})$ is bounded below and let $m=$ $\inf _{X} \varphi$. If $\varphi$ satisfies the $C_{c}$-condition, then we can find $u_{0} \in X$ such that $\varphi\left(u_{0}\right)=$ $\inf _{X} \varphi$.

The next result is known in the literature as the "second deformation theorem" and is one of the main results in critical point theory. First, we introduce some notation. Given $\varphi \in C^{1}(X, \mathbf{R})$ and $c \in \mathbf{R}$, we define

$$
\begin{aligned}
K_{\varphi} & =\left\{u \in X: \varphi^{\prime}(u)=0\right\}, \\
K_{\varphi}^{c} & =\left\{u \in K_{\varphi}: \varphi(u)=c\right\}, \\
\varphi^{c} & =\{u \in X: \varphi(u) \leqslant c\} .
\end{aligned}
$$

Theorem 2. If $\varphi \in C^{1}(X, \mathbf{R}), a \in \mathbf{R}, a<b \leqslant+\infty, \varphi$ satisfies the $C_{c}$-condition for every $c \in[a, b), \varphi$ has no critical values in $(a, b)$ and $\varphi^{-1}(a)$ contains at most a finitely many critical points, then we can find a deformation $h:[0,1] \times\left(\varphi^{b} \backslash K_{\varphi}^{b}\right) \rightarrow \varphi^{b}$ such that

(a) $h\left(1, \varphi^{b} \backslash K_{\varphi}^{b}\right) \subset \varphi^{a}$;

(b) $\left.h(t, \cdot)\right|_{\varphi^{a}}=\left.i d\right|_{\varphi^{a}}$ for all $t \in[0,1]$; 
(c) $\varphi(h(t, u)) \leqslant \varphi(h(s, u))$ for all $t, s \in[0,1]$ with $s \leqslant t$, and for all $u \in \varphi^{b} \backslash K_{\varphi}^{b}$ (that is, the deformation $h$ is " $\varphi$-decreasing").

Remark 1. Note that if $b=+\infty$, then $\varphi^{b} \backslash K_{\varphi}^{b}=X$. The conclusion of Theorem 2 says that $\varphi^{a}$ is a strong deformation retract of $\varphi^{b} \backslash K_{\varphi}^{b}$. A special case of this result, is the so-called "Noncritical Interval Theorem", which says:

"If $\varphi \in C^{1}(X, \mathbf{R})$ satisfies the $C_{c^{-}}$-condition for all $c \in[a, b]$ and $K_{\varphi} \cap$ $\varphi^{-1}[a, b]=\emptyset$, then $\varphi^{a}$ is a strong deformation retract of $\varphi^{b \prime \prime}$.

In critical point theory the notion of linking sets plays a central role:

Definition 3. Let $Y$ be a Hausdorff topological space and $E_{0} \subseteq E$ and $D$ nonempty subsets of $Y$. We say that the pair $\left\{E_{0}, E\right\}$ is "linking" with $D$ in $Y$, if the following conditions hold:

(a) $E_{0} \cap D=\emptyset$;

(b) for any $\gamma \in C(E, Y)$ with $\left.\gamma\right|_{E_{0}}=$ id $\left.\right|_{E_{0}}$ we have $\gamma(E) \cap D \neq \emptyset$.

Using this notion, one can prove a general minimax principle from which follow as special cases the classical results of critical point theory (mountain pass theorem, saddle point theorem, generalized mountain pass theorem). For future use we state the mountain pass theorem.

Theorem 4. Assume that $\varphi \in C^{1}(X, \mathbf{R}), u_{0}, u_{1} \in X,\left\|u_{1}-u_{0}\right\|>r$,

$$
\begin{aligned}
& \max \left\{\varphi\left(u_{0}\right), \varphi\left(u_{1}\right)\right\} \leqslant \inf \left[\varphi(u):\left\|u-u_{0}\right\|=r\right]=m_{r}, \\
& c=\inf _{\gamma \in \Gamma} \max _{0 \leqslant t \leqslant 1} \varphi(\gamma(t)) \text { with } \Gamma=\left\{\gamma \in C([0,1], X): \gamma(0)=u_{0}, \gamma(1)=u_{1}\right\}
\end{aligned}
$$

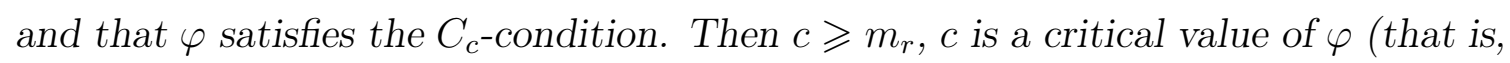
$\left.K_{\varphi}^{c} \neq \emptyset\right)$ and if $c=m_{r}$, then

$$
K_{\varphi}^{c} \cap \partial B_{r}\left(u_{0}\right) \neq \emptyset
$$

with $\partial B_{r}\left(u_{0}\right)=\left\{u \in X:\left\|u-u_{0}\right\|=r\right\}$.

Remark 2. For Theorem 4 the linking sets are

$$
E_{0}=\left\{u_{0}, u_{1}\right\}, \quad E=\left\{(1-t) u_{0}+t u_{1}: 0 \leqslant t \leqslant 1\right\} \text { and } D=\partial B_{r}\left(u_{0}\right) .
$$

For details on these and related issues we refer to Gasinski and Papageorgiou [9].

In our analysis of problem (1), we will make use of the following spaces:

- the Sobolev space $W^{1, p}(\Omega), 1<p<\infty$;

- the Banach space $C^{1}(\bar{\Omega})$;

- the boundary Lebesgue spaces $L^{q}(\partial \Omega), 1 \leqslant q \leqslant \infty$.

By $\|\cdot\|$ we denote the norm on $W^{1, p}(\Omega)$ defined by

$$
\|u\|=\left[\|u\|_{p}^{p}+\|D u\|_{p}^{p}\right]^{1 / p} \text { for all } u \in W^{1, p}(\Omega) .
$$

By $\langle\cdot, \cdot\rangle$ we denote the duality brackets for the pair $\left(W^{1, p}(\Omega)^{*}, W^{1, p}(\Omega)\right)$. The Banach space $C^{1}(\bar{\Omega})$ is an ordered Banach space with positive (order) cone given by

$$
C_{+}=\left\{u \in C^{1}(\bar{\Omega}): u(z) \geqslant 0 \text { for all } z \in \bar{\Omega}\right\} .
$$

This cone has a nonempty interior containing the open set

$$
D_{+}=\left\{u \in C_{+}: u(z)>0 \text { for all } z \in \bar{\Omega}\right\} .
$$

On $\partial \Omega$ we consider the $(N-1)$-dimensional Hausdorff (surface) measure $\sigma(\cdot)$. Using this measure, we can define in the usual way the Lebesgue spaces $L^{q}(\partial \Omega), 1 \leqslant q \leqslant \infty$. 
We know that there exists a unique continuous linear map $\gamma_{0}: W^{1, p}(\Omega) \rightarrow L^{p}(\partial \Omega)$, known as the "trace map", such that

$$
\gamma_{0}(u)=\left.u\right|_{\partial \Omega} \text { for all } u \in W^{1, p}(\Omega) \cap C(\bar{\Omega}) .
$$

So, the trace map extends the notion of "boundary values" to all Sobolev functions. The trace map $\gamma_{0}$ is compact into $L^{q}(\partial \Omega)$ with $q \in\left[1, \frac{N p-p}{N-p}\right)$ if $p<N$ and $q \in[1, \infty)$ if $p \geqslant N$. In what follows, for the sake of notational simplicity we drop the use of the map $\gamma_{0}$. All restrictions of the Sobolev function on $\partial \Omega$ are understood in the sense of traces.

Our hypotheses on the potential function $\xi(\cdot)$ and the boundary coefficient $\beta(\cdot)$ are the following:

$H(\xi): \xi \in L^{\infty}(\Omega)$.

$H(\beta): \beta \in C^{0, \alpha}(\partial \Omega)$ with $\alpha \in(0,1)$ and $\beta(z) \geqslant 0$ for all $z \in \partial \Omega$.

We consider the $C^{1}$-functional $\vartheta: W^{1, p}(\Omega) \rightarrow \mathbf{R}$ defined by

$$
\vartheta(u)=\|D u\|_{p}^{p}+\int_{\Omega} \xi(z)|u|^{p} d z+\int_{\partial \Omega} \beta(z)|u|^{p} d \sigma \text { for all } u \in W^{1, p}(\Omega) .
$$

Let $f_{0}: \Omega \times \mathbf{R} \rightarrow \mathbf{R}$ be a Carathéodory function such that

$$
\left|f_{0}(z, x)\right| \leqslant a_{0}(z)\left(1+|x|^{r-1}\right) \text { for almost all } z \in \Omega \text {, and for all } x \in \mathbf{R},
$$

with $a_{0} \in L^{\infty}(\Omega)_{+}$and $1<r \leqslant p^{*}=\left\{\begin{array}{ll}\frac{N p}{N-p} & \text { if } p<N \\ +\infty & \text { if } p \geqslant N\end{array}\right.$ (the critical Sobolev exponent). We set $F_{0}(z, x)=\int_{0}^{x} f_{0}(z, s) d s$ and consider the $C^{1}$-functional $\varphi_{0}: W^{1, p}(\Omega) \rightarrow$ $\mathbf{R}$ defined by

$$
\varphi_{0}(u)=\frac{1}{p} \vartheta(u)-\int_{\Omega} F_{0}(z, u) d z \text { for all } u \in W^{1, p}(\Omega) .
$$

From Papageorgiou and Rădulescu [18] (subcritical case) and [20] (critical case) we have the following result.

Proposition 5. Assume that $u_{0} \in W^{1, p}(\Omega)$ is a local $C^{1}(\bar{\Omega})$-minimizer of $\varphi_{0}$, that is, there exists $\rho_{0}>0$ such that

$$
\varphi_{0}\left(u_{0}\right) \leqslant \varphi_{0}\left(u_{0}+h\right) \text { for all } h \in C^{1}(\bar{\Omega}) \text { with }\|h\|_{C^{1}(\bar{\Omega})} \leqslant \rho_{0} .
$$

Then $u_{0} \in C^{1, \alpha}(\bar{\Omega})$ for some $\alpha \in(0,1)$ and $u_{0}$ is also a local $W^{1, p}(\Omega)$-minimizer of $\varphi_{0}$, that is, there exists $\rho_{1}>0$ such that

$$
\varphi_{0}\left(u_{0}\right) \leqslant \varphi_{0}\left(u_{0}+h\right) \text { for all } h \in W^{1, p}(\Omega) \text { with }\|h\| \leqslant \rho_{1} .
$$

Let $A: W^{1, p}(\Omega) \rightarrow W^{1, p}(\Omega)^{*}$ be the nonlinear map defined by

$$
\langle A(u), h\rangle=\int_{\Omega}|D u|^{p-2}(D u, D h)_{\mathbf{R}^{N}} d z \text { for all } u, h \in W^{1, p}(\Omega) .
$$

The following well-known result summarizes the main properties of the map $A(\cdot)$ (see, for example, Motreanu, Motreanu and Papageorgiou [15, p. 40]).

Proposition 6. The map $A: W^{1, p}(\Omega) \rightarrow W^{1, p}(\Omega)^{*}$ is bounded (maps bounded sets to bounded sets), continuous, monotone (thus maximal monotone, too) and of type $(S)_{+}$that is,

$$
\text { " } u_{n} \stackrel{w}{\rightarrow} u \text { in } W^{1, p}(\Omega) \text { and } \limsup _{n \rightarrow \infty}\left\langle A\left(u_{n}\right), u_{n}-u\right\rangle \leqslant 0 \Rightarrow u_{n} \rightarrow u \text { in } W^{1, p}(\Omega) . "
$$


We will also use some facts about the spectrum of the differential operator $u \mapsto$ $-\Delta_{p} u+\xi(z) u$ with Robin boundary condition.

So, we consider the following nonlinear eigenvalue problem:

$$
\left\{\begin{array}{l}
-\Delta_{p} u(z)+\xi(z)|u(z)|^{p-2} u(z)=\hat{\lambda}|u(z)|^{p-2} u(z), \\
\frac{\partial u}{\partial n_{p}}+\beta(z)|u|^{p-2} u=0 \text { on } \partial \Omega .
\end{array}\right.
$$

By an eigenvalue, we mean any $\hat{\lambda} \in \mathbf{R}$ for which problem (2) has a nontrivial solution $\hat{u} \in W^{1, p}(\Omega)$, known as an eigenfunction corresponding to the eigenvalue $\hat{\lambda}$. From Papageorgiou and Rădulescu [20], we know that $\hat{u} \in L^{\infty}(\Omega)$ and so we can apply Theorem 2 of Lieberman [14] and infer that $\hat{u} \in C^{1}(\bar{\Omega})$.

From Mugnai and Papageorgiou [16] and Papageorgiou and Rădulescu [18], we know that problem (2) admits a smallest eigenvalue $\hat{\lambda}_{1} \in \mathbf{R}$ which has the following properties:

- $\hat{\lambda}_{1}$ is isolated in the spectrum $\sigma_{0}(p)$ of (2) (that is, we can find $\epsilon>0$ such that $\left.\left(\hat{\lambda}_{1}, \hat{\lambda}_{1}+\epsilon\right) \cap \sigma_{0}(p)=\emptyset\right)$.

- $\hat{\lambda}_{1}$ is simple (that is, if $\hat{u}, \hat{v}$ are eigenfunctions corresponding to $\hat{\lambda}_{1}$, then $\hat{u}=\eta \hat{v}$ with $\eta \in \mathbf{R} \backslash\{0\})$.

$$
\text { - } \hat{\lambda}_{1}=\inf \left\{\frac{\vartheta(u)}{\|u\|_{p}^{p}}: u \in W^{1, p}(\Omega), u \neq 0\right\} \text {. }
$$

The infimum in (3) is realized on the one-dimensional eigenspace corresponding to $\hat{\lambda}_{1}$. The above properties of $\hat{\lambda}_{1}$ imply that the eigenfunctions corresponding to $\hat{\lambda}_{1}$ do not change sign. Let $\hat{u}_{1}$ be the $L^{p}$-normalized (that is, $\left\|\hat{u}_{1}\right\|_{p}=1$ ) positive eigenfunction corresponding to $\hat{\lambda}_{1}$. As we already mentioned, the nonlinear regularity theory implies that $\hat{u}_{1} \in C_{+}$. In fact, the nonlinear maximum principle (see, for example, Gasinski and Papageorgiou [9, p. 738]), implies that $\hat{u}_{1} \in D_{+}$. An eigenfunction $\hat{u}$ which corresponds to an eigenvalue $\hat{\lambda} \neq \hat{\lambda}_{1}$ is nodal (that is, sign changing). Since the spectrum $\sigma_{0}(p)$ of (2) is closed and $\hat{\lambda}_{1}$ is isolated, the second eigenvalue $\hat{\lambda}_{2}$ is well-defined by

$$
\hat{\lambda}_{2}=\min \left\{\hat{\lambda} \in \sigma_{0}(p): \hat{\lambda}>\hat{\lambda}_{1}\right\} .
$$

To produce additional eigenvalues, we employ the Ljusternik-Schnirelmann minimax scheme, which generates a whole nondecreasing sequence $\left\{\hat{\lambda}_{k}\right\}_{k \in \mathbf{N}}$ of eigenvalues of (2) such that $\hat{\lambda}_{k} \rightarrow+\infty$. These eigenvalues are known as "variational eigenvalues" and depending on the index used in the execution of the Ljusternik-Schirelmann minimax scheme, we generate different sequences of variational eigenvalues. We do not know if these sequences coincide and if they exhaust the spectrum $\sigma_{0}(p)$. This is the case if $p=2$ (linear eigenvalue problem) or if $N=1$ (ordinary differential equation). Moreover, we know that all these sequences of variational eigenvalues coincide in the first two elements $\hat{\lambda}_{1}$ and $\hat{\lambda}_{2}$, which are given by (3) and (4). In fact, for $\hat{\lambda}_{2}$ we have a useful minimax characterization. So, let

$$
\begin{aligned}
\partial B_{1}^{L^{p}} & =\left\{u \in L^{p}(\Omega):\|u\|_{p}=1\right\} \\
M & =W^{1, p}(\Omega) \cap \partial B_{1}^{L^{p}} \\
\hat{\Gamma} & =\left\{\hat{\gamma} \in C([-1,1], M): \hat{\gamma}(-1)=-\hat{u}_{1}, \hat{\gamma}(1)=\hat{u}_{1}\right\} .
\end{aligned}
$$

Using these items we can get the following minimax characterization of $\hat{\lambda}_{2}$ (see $[16,18])$. 
Proposition 7. $\hat{\lambda}_{2}=\inf _{\hat{\gamma} \in \hat{\Gamma}} \max _{-1 \leqslant t \leqslant 1} \vartheta(\hat{\gamma}(t))$.

Here we use the sequence of variational eigenvalues generated by the LjusternikSchnirelmann scheme when as the index we use the Fadell-Rabinowitz cohomological index (see [8]).

Finally, let us recall some basic definitions and facts from critical groups which we will use in the sequel. So, let $\left(Y_{1}, Y_{2}\right)$ be a topological pair such that $Y_{2} \subseteq Y_{1} \subseteq X$. By $H_{k}\left(Y_{1}, Y_{2}\right), k \in \mathbf{N}_{0}$, we denote the $k t h$ relative singular homology group with integer coefficients for the pair $\left(Y_{1}, Y_{2}\right)$. If $\varphi \in C^{1}(X, \mathbf{R})$ and $u \in K_{\varphi}^{c}$ is isolated, then the critical groups of $\varphi$ at $u$ are defined by

$$
C_{k}(\varphi, u)=H_{k}\left(\varphi^{c} \cap U, \varphi^{c} \cap U \backslash\{0\}\right) \text { for all } k \in \mathbf{N}_{0},
$$

with $U$ being a neighborhood of $u$ such that $K_{\varphi} \cap \varphi^{c} \cap U=\{u\}$. The excision property of singular homology implies that this definition is independent of the choice of the neighborhood $U$.

Suppose that $\varphi$ satisfies the $C$-condition and $\inf \varphi\left(K_{\varphi}\right)>-\infty$. Let $c<\inf \varphi\left(K_{\varphi}\right)$. The critical groups of $\varphi$ at infinity are defined by

$$
C_{k}(\varphi, \infty)=H_{k}\left(X, \varphi^{c}\right) \text { for all } k \in \mathbf{N}_{0} .
$$

This definition is independent of the choice of the level $c<\inf \varphi\left(K_{\varphi}\right)$. Indeed, if $c_{0}<c<\inf \varphi\left(K_{\varphi}\right)$, then by the Noncritical Interval Theorem (see Remark 1), we have that

$\varphi^{c_{0}}$ is a strong deformation retract of $\varphi^{c}$,

$\Rightarrow H_{k}\left(X, \varphi^{c}\right)=H_{k}\left(X, \varphi^{c_{0}}\right)$ for all $k \in \mathbf{N}_{0}$,

(see Motreanu, Motreanu and Papageorgiou [15, p. 145]).

We introduce the following quantities

$$
\begin{aligned}
& M(t, u)=\sum_{k \geqslant 0} \operatorname{rank} C_{k}(\varphi, u) t^{k} \quad \text { for all } t \in \mathbf{R}, \text { all } u \in K_{\varphi}, \\
& P(t, \infty)=\sum_{k \geqslant 0} \operatorname{rank} C_{k}(\varphi, \infty) t^{k} \quad \text { for all } t \in \mathbf{R} .
\end{aligned}
$$

Then the "Morse relation" says that

$$
\sum_{u \in K_{\varphi}} M(t, u)=P(t, \infty)+(1+t) Q(t) \text { for all } t \in \mathbf{R},
$$

with $Q(t)=\sum_{k \geqslant 0} \beta_{k} t^{k}$ being a formal series in $t \in \mathbf{R}$ with nonnegative integer coefficients $\beta_{k}$.

Now let

$$
V_{p}=\left\{u \in W^{1, p}(\Omega): \int_{\Omega} \hat{u}_{1}^{p-1} u d z=0\right\} .
$$

We have the following direct sum decomposition

$$
W^{1, p}(\Omega)=\mathbf{R} \hat{u}_{1} \oplus V_{p}
$$

We define

$$
\hat{\lambda}(p)=\inf \left\{\frac{\vartheta(u)}{\|u\|_{p}^{p}}: u \in V_{p}, u \neq 0\right\} .
$$

Proposition 8. If hypotheses $H(\xi), H(\beta)$ hold, then $\hat{\lambda}_{1}<\hat{\lambda}(p) \leqslant \hat{\lambda}_{2}$. 
Proof. Note that (3) and (6) imply that $\hat{\lambda}_{1} \leqslant \hat{\lambda}(p)$. Suppose that $\hat{\lambda}_{1}=\hat{\lambda}(p)$. Consider a sequence $\left\{u_{n}\right\}_{n \geqslant 1} \subseteq V_{p}$ such that

$$
\left\|u_{n}\right\|_{p}=1 \text { for all } n \in \mathbf{N} \text { and } \vartheta\left(u_{n}\right) \downarrow \hat{\lambda}(p)=\hat{\lambda}_{1} \text { as } n \rightarrow \infty \text {. }
$$

So, the sequence $\left\{u_{n}\right\}_{n \geqslant 1} \subseteq W^{1, p}(\Omega)$ is bounded and thus, by passing to a suitable subsequence if necessary, we may assume that

$$
u_{n} \stackrel{w}{\rightarrow} u \text { in } W^{1, p}(\Omega) \text { and } u_{n} \rightarrow u \text { in } L^{p}(\Omega) \text { and in } L^{p}(\partial \Omega) .
$$

Since the functional $\vartheta(\cdot)$ is sequentially weakly lower semicontinuous, it follows from (7) and (8) that

$$
\begin{aligned}
& \vartheta(u) \leqslant \hat{\lambda}(p)=\hat{\lambda}_{1}, \quad\|u\|_{p}=1, \quad u \in V_{p}, \\
& \Rightarrow \vartheta(u)=\hat{\lambda}(p)=\hat{\lambda}_{1} \quad(\text { see }(6)), \\
& \Rightarrow u=\eta \hat{u}_{1} \text { with } \eta \in \mathbf{R} \backslash\{0\} .
\end{aligned}
$$

If $\eta \neq 0$, then $u \notin V_{p}$, a contradiction (see (8)). If $\eta=0$, then $u=0$, a contradiction since $\|u\|_{p}=1$ (see (8)). So, we have proved that $\hat{\lambda}_{1}<\hat{\lambda}(p)$.

Next, we show that $\hat{\lambda}(p) \leqslant \hat{\lambda}_{2}$. Arguing by contradiction, suppose that $\hat{\lambda}_{2}<\hat{\lambda}(p)$. Then from Proposition 7 we see that we can find $\hat{\gamma}_{0} \in \hat{\Gamma}$ such that

$$
\vartheta\left(\hat{\gamma}_{0}(t)\right)<\hat{\lambda}(p) \text { for all } t \in[-1,1] \text {. }
$$

Let $\tau:[-1,1] \rightarrow \mathbf{R}$ be defined by

$$
\tau(t)=\int_{\Omega} \hat{u}_{1}^{p-1} \hat{\gamma}_{0}(t) d z \text { for all } t \in[-1,1] .
$$

Evidently, $\tau(\cdot)$ is continuous and we have

$$
\left.\tau(-1)=-1, \tau(1)=1 \quad \text { (recall that }\left\|\hat{u}_{1}\right\|_{p}=1\right) .
$$

So, by Bolzano's theorem, we can find $t_{0} \in(-1,1)$ such that

$$
\begin{aligned}
& \tau\left(t_{0}\right)=\int_{\Omega} \hat{u}_{1}^{p-1} \hat{\gamma}_{0}\left(t_{0}\right) d z=0, \\
& \Rightarrow \hat{\gamma}_{0}\left(t_{0}\right) \in V_{p} \\
& \Rightarrow \hat{\lambda}(p) \leqslant \vartheta\left(\hat{\gamma}_{0}\left(t_{0}\right)\right) \quad(\text { see }(6)) .
\end{aligned}
$$

Comparing (10) and (11), we reach a contradiction. Therefore we obtain

$$
\hat{\lambda}_{1}<\hat{\lambda}(p) \leqslant \hat{\lambda}_{2} \text {. }
$$

The proof is now complete.

Remark 3. If $p=2$, then $\hat{\lambda}(2)=\hat{\lambda}_{2}$.

Now, let $\lambda>\hat{\lambda}_{2}, \lambda \notin \sigma_{0}(p)$ and consider the $C^{1}$-functional $\psi_{\lambda}: W^{1, p}(\Omega) \rightarrow \mathbf{R}$ defined by

$$
\psi_{\lambda}(u)=\frac{1}{p} \vartheta(u)-\frac{\lambda}{p}\|u\|_{p}^{p} \text { for all } u \in W^{1, p}(\Omega) .
$$

Proposition 9. If hypotheses $H(\xi), H(\beta)$ hold, then $C_{0}\left(\psi_{\lambda}, 0\right)=C_{1}\left(\psi_{\lambda}, 0\right)=0$.

Proof. Let $D=\left\{u \in W^{1, p}(\Omega): \vartheta(u)<\lambda\|u\|_{p}^{p}\right\}$. Evidently, $D \subseteq W^{1, p}(\Omega)$ is open and $\pm \hat{u}_{1} \in D$.

Claim 1. The set $D$ is path-connected. 
Let $u \in D$ and let $E_{u}$ be the path-component of $D$ containing $u$. We set

$$
m_{u}=\inf \left\{\frac{\vartheta(v)}{\|v\|_{p}^{p}}: v \in E_{u}\right\}<\lambda .
$$

Let $\left\{v_{n}\right\}_{n \geqslant 1} \subseteq E_{u}$ be such that

$$
\frac{\vartheta\left(v_{n}\right)}{\left\|v_{n}\right\|_{p}^{p}} \downarrow m_{u} \text { as } n \rightarrow \infty .
$$

The $p$-homogeneity of $\vartheta(\cdot)$, allows us to assume that

$$
\left\|v_{n}\right\|_{p}=1 \text { for all } n \in \mathbf{N} \text {. }
$$

Then it follows from (13) and (14) that $\left\{v_{n}\right\}_{n \geqslant 1} \subseteq W^{1, p}(\Omega)$ is bounded. Recall that

$$
M=W^{1, p}(\Omega) \cap \partial B_{1}^{L^{p}}=\left\{u \in W^{1, p}(\Omega):\|u\|_{p}=1\right\} .
$$

Employing the Ekeland variational principle (see, for example, Gasinski and Papageorgiou $\left[9\right.$, p. 579]), we can find $\left\{y_{n}\right\}_{n \geqslant 1} \subset \overline{E_{u} \cap M}$ such that

$$
\left\{\begin{array}{l}
\vartheta\left(y_{n}\right) \leqslant \vartheta\left(v_{n}\right) \leqslant m_{u}+\frac{1}{n^{2}}, \quad\left\|y_{n}-v_{n}\right\|<\frac{1}{n}, \\
\vartheta\left(y_{n}\right) \leqslant \vartheta(v)+\frac{1}{n^{2}}\left\|v-y_{n}\right\| \text { for all } v \in \overline{E_{u} \cap M}, \text { all } n \in \mathbf{N} .
\end{array}\right.
$$

Suppose that $y_{n} \in \partial \overline{\left(E_{u} \cap M\right)}$ for infinitely many $n \in \mathbf{N}$ (to simplify things we assume that this holds for every $n \in \mathbf{N}$ ). Then Lemma 3.5(iii) of Cuesta, de Figueiredo and Gossez [6] implies that

$$
\vartheta\left(y_{n}\right)=\lambda \leqslant \vartheta\left(v_{n}\right) \leqslant m_{u}+\frac{1}{n^{2}}<\lambda \text { for all large enough } n \in \mathbf{N}(\text { see }(15)),
$$

a contradiction. This means that

$$
y_{n} \in E_{u} \cap M \text { for all } n \in \mathbf{N} .
$$

Then it follows from (15) that

$$
\left(\left.\vartheta\right|_{M}\right)^{\prime}\left(y_{n}\right) \rightarrow 0 \text { as } n \rightarrow \infty .
$$

As in the proof of Proposition 5 of Papageorgiou and Rădulescu [18] (see Claim 1 in that proof), we can see that

$$
\left.\vartheta\right|_{M} \text { satisfies the C-condition. }
$$

Then from (16), (17) and by passing to a suitable subsequence if necessary, we see that we may assume that

$$
\begin{aligned}
& y_{n} \rightarrow y \text { in } W^{1, p}(\Omega) \text { as } n \rightarrow \infty \\
& \Rightarrow y \in \overline{E_{u} \cap M} \text { and } \vartheta(y)=m_{u}<\lambda, \\
& \Rightarrow y \in E_{u} \cap M \text { (as before, using Lemma 3.5(iii) in Cuesta, } \\
& \text { de Figueiredo and Gossez [6]). }
\end{aligned}
$$

Hence to prove the claim, it suffices to connect $y$ and $\hat{u}_{1}$ with a path staying in $D$ (see Dugundji [7, p. 115]). First, suppose that $y \leqslant 0$. Then $y=-\hat{u}_{1}$ (see (3)) and the desired path is provided by Proposition 7 (recall $\lambda>\hat{\lambda}_{2}$ ). Now, suppose that $y \geqslant 0$. Then $y=\hat{u}_{1}$. Therefore, we may assume that

$$
y^{+}, y^{-} \neq 0 \text {. }
$$

We set

$$
e_{t}=\frac{y^{+}-(1-t) y^{-}}{\left\|y^{+}-(1-t) y^{-}\right\|_{p}} \in M \text { for all } t \in[0,1]
$$


From (16) and (18), we have

$$
\langle A(y), h\rangle+\int_{\Omega} \xi(z)|y|^{p-2} y h d z+\int_{\partial \Omega} \beta(z)|y|^{p-2} y h d \sigma=m_{u} \int_{\Omega}|y|^{p-2} y h d z
$$

for all $h \in W^{1, p}(\Omega)$.

In (19) we first choose $h=y^{+} \in W^{1, p}(\Omega)$ and then we choose $h=-y^{-} \in W^{1, p}(\Omega)$.

We obtain

$$
\vartheta\left(y^{+}\right)=m_{u}\left\|y^{+}\right\|_{p}^{p} \quad \text { and } \quad \vartheta\left(y^{-}\right)=m_{u}\left\|y^{-}\right\|_{p}^{p}
$$

Since $y^{+}$and $y^{-}$have disjoint interior supports, it follows that

$$
\begin{aligned}
& \vartheta\left(e_{t}\right)=m_{u}\left\|e_{t}\right\|_{p}^{p}=m_{u} \text { for all } t \in[0,1], \\
& \Rightarrow e_{t} \in D \text { for all } t \in[0,1] .
\end{aligned}
$$

Note that

$$
e_{0}=\frac{y}{\|y\|_{p}}=y \quad\left(\text { recall } y \in E_{u} \cap M\right) \text { and } e_{1}=\frac{y^{+}}{\left\|y^{+}\right\|_{p}}=\hat{u}_{1} .
$$

Therefore $t \mapsto e_{t}$ is the desired path in $D$. This proves Claim 1 .

If $e \in D$, then from the claim we have

$$
H_{0}(D, e)=0 \quad \text { (see Motreanu, Motreanu and Papageorgiou [15, p. 152]) }
$$

Consider the 0 -sublevel set of $\psi_{\lambda}$

$$
\psi_{\lambda}^{0}=\left\{u \in W^{1, p}(\Omega): \psi_{\lambda}(u) \leqslant 0\right\} .
$$

Since $\psi_{\lambda}(\cdot)$ is $p$-homogeneous, it follows that

$$
\psi_{\lambda}^{0} \text { is contractible, }
$$

$$
\Rightarrow H_{k}\left(\psi_{\lambda}^{0}, e\right)=0, \forall k \in \mathbf{N}_{0} \text { (see Motreanu, Motreanu and }
$$

Papageorgiou [15, p. 147]).

Let $\epsilon>0$ be small. Theorem 2 (the second deformation theorem) implies that

$$
\psi_{\lambda}^{0} \backslash\{0\} \text { and } \psi_{\lambda}^{-\epsilon} \text { are homotopy equivalent. }
$$

Also, let

$$
\dot{\psi}_{\lambda}^{0}=\left\{u \in W^{1, p}(\Omega): \psi_{\lambda}(u)<0\right\}=D .
$$

Since $K_{\psi_{\lambda}}=\{0\}$ (recall that $\lambda \notin \sigma_{0}(p)$ ), from Granas and Dugundji [11, p. 407], we have

$$
\dot{\psi}_{\lambda}^{0}=D \text { and } \psi_{\lambda}^{-\epsilon} \text { are homotopy equivalent. }
$$

From (22) and (23) we infer that

$$
\begin{aligned}
& \psi_{\lambda}^{0} \backslash\{0\} \text { and } D \text { are homotopy equivalent, } \\
& \Rightarrow H_{k}\left(\psi_{\lambda}^{0} \backslash\{0\}, e\right)=H_{k}(D, e) \text { for all } k \in \mathbf{N}_{0}, \\
& \Rightarrow H_{0}\left(\psi_{\lambda}^{0} \backslash\{0\}, e\right)=0 \quad(\text { see }(20)) .
\end{aligned}
$$

We consider the following long exact reduced singular homology sequence

$$
\begin{gathered}
\ldots \rightarrow H_{k}\left(\psi_{\lambda}^{0} \backslash\{0\}, e\right) \rightarrow H_{k}\left(\psi_{\lambda}^{0}, e\right)=0 \stackrel{i_{*}}{\rightarrow} H_{k}\left(\psi_{\lambda}^{0}, \psi_{\lambda}^{0} \backslash\{0\}\right)=C_{k}\left(\psi_{\lambda}, 0\right) \\
\stackrel{\partial_{*}}{\rightarrow} H_{k-1}\left(\psi_{\lambda}^{0} \backslash\{0\}, e\right) \rightarrow \cdots
\end{gathered}
$$


with $i_{*}$ being the group homomorphism corresponding to the inclusion map $i$ and $\partial_{*}$ is the boundary homomorphism. By the exactness of $(25)$ we have that $\partial_{*}$ is an isomorphism between $C_{1}\left(\psi_{\lambda}, 0\right)$ and a subgroup of $H_{0}\left(\psi_{\lambda}^{0} \backslash\{0\}, e\right)$ and so

$$
C_{1}\left(\psi_{\lambda}, 0\right)=0 \quad(\text { see }(24)) \text {. }
$$

It follows from (25) and (26) that

$$
C_{0}\left(\psi_{\lambda}, 0\right)=0
$$

The proof is now complete.

\section{Resonance at a nonprincipal eigenvalue}

In this section we prove two existence theorems for the case when the equation is resonant with respect to a nontrivial eigenvalue $\hat{\lambda}_{m}$.

For the first existence theorem, the hypotheses on the reaction term are the following:

$H_{1}: f: \Omega \times \mathbf{R} \rightarrow \mathbf{R}$ is a Carathéodory function such that $f(z, 0)=0$ for almost all $z \in \Omega$ and

(i) for every $\rho>0$, there exists $a_{\rho} \in L^{\infty}(\Omega)_{+}$such that

$$
|f(z, x)| \leqslant a_{\rho}(z) \text { for almost all } z \in \Omega \text {, and for all }|x| \leqslant \rho ;
$$

(ii) there exists $m \in \mathbf{N}, m \geqslant 2$ such that

$$
\lim _{x \rightarrow \pm \infty} \frac{f(z, x)}{|x|^{p-2} x}=\hat{\lambda}_{m} \text { uniformly for almost all } z \in \Omega ;
$$

(iii) if $F(z, x)=\int_{0}^{x} f(z, s) d s$, then $\lim _{x \rightarrow \pm \infty}[f(z, x) x-p F(z, x)]=+\infty$ uniformly for almost all $z \in \Omega$;

(iv) there exist $\eta \in L^{\infty}(\Omega), \hat{\lambda}_{1} \leqslant \eta(z)$ for almost all $z \in \Omega, \eta \not \equiv \hat{\lambda}_{1}, \hat{\eta}<\hat{\lambda}(p)$ and $\delta>0$ such that

$$
\frac{1}{p} \eta(z)|x|^{p} \leqslant F(z, x) \leqslant \frac{1}{p} \hat{\eta}|x|^{p} \text { for almost all } z \in \Omega \text {, and for all }|x| \leqslant \delta .
$$

Let $\varphi: W^{1, p}(\Omega) \rightarrow \mathbf{R}$ be the energy (Euler) functional for problem (1) defined by

$$
\varphi(u)=\frac{1}{p} \vartheta(u)-\int_{\Omega} F(z, u) d z \text { for all } u \in W^{1, p}(\Omega) .
$$

Evidently, $\varphi \in C^{1}\left(W^{1, p}(\Omega)\right)$.

Proposition 10. If hypotheses $H(\xi), H(\beta), H_{1}$ hold, then the functional $\varphi$ satisfies the $C$-condition.

Proof. Let $\left\{u_{n}\right\}_{n \geqslant 1} \subseteq W^{1, p}(\Omega)$ be a sequence such that

$$
\begin{aligned}
& \left|\varphi\left(u_{n}\right)\right| \leqslant M_{1} \text { for some } M_{1}>0, \text { and for all } n \in \mathbf{N}, \\
& \left(1+\left\|u_{n}\right\|\right) \varphi^{\prime}\left(u_{n}\right) \rightarrow 0 \text { in } W^{1, p}(\Omega)^{*} \text { as } n \rightarrow \infty .
\end{aligned}
$$

From (28) we have

$$
\left.\left|\left\langle A\left(u_{n}\right), h\right\rangle+\int_{\Omega} \xi(z)\right| u_{n}\right|^{p-2} u_{n} h d z+\int_{\partial \Omega} \beta(z)\left|u_{n}\right|^{p-2} u_{n} h d \sigma-\int_{\Omega} f\left(z, u_{n}\right) h d z \mid
$$

$(29) \leqslant \frac{\epsilon_{n}\|h\|}{1+\left\|u_{n}\right\|}$ for all $h \in W^{1, p}(\Omega)$, with $\epsilon_{n} \rightarrow 0^{+}$. 
In $(29)$ we choose $h=u_{n} \in W^{1, p}(\Omega)$. Then

$$
-\vartheta\left(u_{n}\right)+\int_{\Omega} f\left(z, u_{n}\right) u_{n} d z \leqslant \epsilon_{n} \text { for all } n \in \mathbf{N} .
$$

From (27) we have

$$
\vartheta\left(u_{n}\right)-\int_{\Omega} p F\left(z, u_{n}\right) d z \leqslant p M_{1} \text { for all } n \in \mathbf{N} .
$$

Adding (30) and (31), we obtain

$$
\int_{\Omega}\left[f\left(z, u_{n}\right) u_{n}-p F\left(z, u_{n}\right)\right] d z \leqslant M_{2} \text { for some } M_{2}>0 \text {, and for all } n \in \mathbf{N} \text {. }
$$

Claim 2. $\left\{u_{n}\right\}_{n \geqslant 1} \subseteq W^{1, p}(\Omega)$ is bounded.

Arguing by contradiction, suppose that Claim 2 is not true. By passing to a suitable subsequence if necessary, we can say that

$$
\left\|u_{n}\right\| \rightarrow \infty \text {. }
$$

Let $y_{n}=\frac{u_{n}}{\left\|u_{n}\right\|}$ for all $n \in \mathbf{N}$. Then $\left\|y_{n}\right\|=1$ and so we may assume that

$$
y_{n} \stackrel{w}{\rightarrow} y \text { in } W^{1, p}(\Omega) \text { and } y_{n} \rightarrow y \text { in } L^{p}(\Omega) \text { and in } L^{p}(\partial \Omega) .
$$

From (29) we have

$$
\begin{aligned}
& \left.\left|\left\langle A\left(y_{n}\right), h\right\rangle+\int_{\Omega} \xi(z)\right| y_{n}\right|^{p-2} y_{n} h d z+\int_{\partial \Omega} \beta(z)\left|y_{n}\right|^{p-2} y_{n} h d \sigma-\int_{\Omega} \frac{f\left(z, u_{n}\right)}{\left\|u_{n}\right\|^{p-1}} h d z \mid \\
& \leqslant \frac{\epsilon_{n}\|h\|}{\left(1+\left\|u_{n}\right\|\right)\left\|u_{n}\right\|^{p-1}} \text { for all } n \in \mathbf{N} .
\end{aligned}
$$

From hypotheses $H_{1}(\mathrm{i})$,(ii) we see that

$$
|f(z, x)| \leqslant c_{1}\left(1+|x|^{p-1}\right) \text { for almost all } z \in \Omega \text {, and for all } x \in \mathbf{R} \text {, with } c_{1}>0 \text {, }
$$

$$
\Rightarrow\left\{\frac{f\left(\cdot, u_{n}(\cdot)\right)}{\left\|u_{n}\right\|^{p-1}}\right\}_{n \geqslant 1} \subseteq L^{p^{\prime}}(\Omega)\left(\frac{1}{p}+\frac{1}{p^{\prime}}=1\right) \text { is bounded. }
$$

In (35) we choose $h=y_{n}-y \in W^{1, p}(\Omega)$, pass to the limit as $n \rightarrow \infty$ and use (33), (34), (36). We obtain

$$
\begin{aligned}
& \lim _{n \rightarrow \infty}\left\langle A\left(y_{n}\right), y_{n}-y\right\rangle=0, \\
& \left.\Rightarrow y_{n} \rightarrow y \text { in } W^{1, p}(\Omega) \quad \text { (see Proposition } 6\right) \text { and so }\|y\|=1 .
\end{aligned}
$$

From (37) we see that $y \neq 0$. Let $E=\{z \in \Omega: y(z) \neq 0\}$. If by $|\cdot|_{N}$ we denote the Lebesgue measure on $\mathbf{R}^{N}$, then $|E|_{N}>0$. We have

$$
\left|u_{n}(z)\right| \rightarrow+\infty \text { for almost all } z \in E \text {, }
$$$$
\Rightarrow f\left(z, u_{n}(z)\right) u_{n}(z)-p F\left(z, u_{n}(z)\right) \rightarrow+\infty \text { for almost all } z \in \Omega
$$

(see hypothesis $H_{1}(\mathrm{iii})$ ).

From (38), hypothesis $H_{1}$ (iii) and Fatou's lemma, we have

$$
\int_{E}\left[f\left(z, u_{n}\right) u_{n}-p F\left(z, u_{n}\right)\right] d z \rightarrow+\infty \text { as } n \rightarrow \infty .
$$

On the other hand, hypotheses $H_{1}$ (i),(iii) imply that we can find $c_{2}>0$ such that

$$
-c_{2} \leqslant f(z, x) x-p F(z, x) \text { for almost all } z \in \Omega \text {, and for all } x \in \mathbf{R} \text {. }
$$


Then we have

$$
\begin{aligned}
& \int_{\Omega}\left[f\left(z, u_{n}\right) u_{n}-p F\left(z, u_{n}\right)\right] d z \\
& =\int_{E}\left[f\left(z, u_{n}\right) u_{n}-p F\left(z, u_{n}\right)\right] d z+\int_{\Omega \backslash E}\left[f\left(z, u_{n}\right) u_{n}-p F\left(z, u_{n}\right)\right] d z \\
& \geqslant \int_{E}\left[f\left(z, u_{n}\right) u_{n}-p F\left(z, u_{n}\right)\right] d z-c_{2}|\Omega|_{N} \quad(\text { see }(40)), \\
& \left.\Rightarrow \int_{\Omega}\left[f\left(z, u_{n}\right) u_{n}-p F\left(z, u_{n}\right)\right] d z \rightarrow+\infty \text { as } n \rightarrow \infty \text { (see }(39)\right) .
\end{aligned}
$$

This contradicts (32). So, we have proved Claim 2.

Because of Claim 2, at least for a subsequence, we may assume that

$$
u_{n} \stackrel{w}{\rightarrow} u \text { in } W^{1, p}(\Omega) \text { and } u_{n} \rightarrow u \text { in } L^{p}(\Omega) \text { and } L^{p}(\partial \Omega) .
$$

Note that $\left\{f\left(\cdot, u_{n}(\cdot)\right)\right\}_{n \geqslant 1} \subseteq L^{p^{\prime}}(\Omega)$ is bounded. So, if in (29) we choose $h=u_{n}-u \in$ $W^{1, p}(\Omega)$, pass to the limit as $n \rightarrow \infty$ and use (41), then

$$
\begin{aligned}
& \lim _{n \rightarrow \infty}\left\langle A\left(u_{n}\right), u_{n}-u\right\rangle=0 \\
& \Rightarrow u_{n} \rightarrow u \text { in } W^{1, p}(\Omega) \quad(\text { see Proposition } 6) \\
& \Rightarrow \varphi \text { satisfies the C-condition. }
\end{aligned}
$$

The proof is now complete.

Proposition 11. If hypotheses $H(\xi), H(\beta), H_{1}$ hold, then $C_{1}(\varphi, 0) \neq 0$.

Proof. We consider the direct sum decomposition

$$
W^{1, p}(\Omega)=\mathbf{R} \hat{u}_{1} \oplus V_{p}
$$

For small $|t| \in(0,1)$ we have

$$
|t| \hat{u}_{1}(z) \in(0, \delta] \quad \text { for all } z \in \bar{\Omega} \quad\left(\text { recall } \hat{u}_{1} \in D_{+}\right) .
$$

Here $\delta>0$ is as in hypothesis $H_{1}(\mathrm{iv})$. Then

$$
\begin{aligned}
\varphi\left(t \hat{u}_{1}\right) & =\frac{|t|^{p}}{p} \vartheta\left(\hat{u}_{1}\right)-\int_{\Omega} F\left(z, t \hat{u}_{1}\right) d z \\
& \leqslant \frac{|t|^{p}}{p}\left[\vartheta\left(\hat{u}_{1}\right)-\int_{\Omega} \eta(z) \hat{u}_{1}^{p} d z\right] \\
& \text { (see hypothesis } \left.H_{1}(i v) \text { and recall that }\left\|\hat{u}_{1}\right\|_{p}=1\right) \\
& =\frac{|t|^{p}}{p} \int_{\Omega}\left[\hat{\lambda}_{1}-\eta(z)\right] \hat{u}_{1}^{p} d z \\
& <0 \text { for all small }|t| \in(0,1) \quad\left(\text { recall that } \hat{u}_{1} \in D_{+}\right) .
\end{aligned}
$$

Hypotheses $H_{1}$ imply that given $r \in\left(p, p^{*}\right)$, we can find $c_{3}=c_{3}(r)>0$ such that

$$
F(z, x) \leqslant \frac{\hat{\eta}}{p}|x|^{p}+c_{3}|x|^{r} \text { for almost all } z \in \Omega, \text { all } x \in \mathbf{R} .
$$


Then for all $v \in V_{p}$, we have

$$
\begin{aligned}
\varphi(v) & =\frac{1}{p} \vartheta(v)-\int_{\Omega} F(z, v) d z \\
& \geqslant \frac{1}{p}\left[\vartheta(v)-\hat{\eta}\|v\|_{p}^{p}\right]-c_{4}\|v\|^{r} \text { for some } c_{4}>0 \quad(\text { see }(44)) \\
& \left.\geqslant c_{5}\|v\|^{p}-c_{4}\|v\|^{r} \text { for some } c_{5}>0 \quad \text { (recall that } \hat{\eta}<\hat{\lambda}(p)\right) .
\end{aligned}
$$

Since $p<r$, we can find small $\delta_{1} \in(0,1)$ such that

$$
\varphi(v)>0=\varphi(0) \text { for all } 0<\|v\| \leqslant \delta_{1} .
$$

Relations (43) and (45) imply that $\varphi$ has a local linking at the origin with respect to the decomposition $\mathbf{R} \hat{u}_{1} \oplus V_{p}$. So, Corollary 6.88 of Motreanu, Motreanu and Papageorgiou [15, p. 172] implies that $C_{1}(\varphi, 0) \neq 0$.

Proposition 12. If hypotheses $H(\xi), H(\beta), H_{1}$ hold, then $C_{0}(\varphi, \infty)=C_{1}(\varphi, \infty)=$ 0 and $C_{m}(\varphi, \infty) \neq 0$.

Proof. Let $\lambda \in\left(\hat{\lambda}_{m}, \hat{\lambda}_{m+1}\right) \backslash \sigma_{0}(p)$ and as before (see Section 2), let $\psi_{\lambda}: W^{1, p}(\Omega) \rightarrow$ $\mathbf{R}$ be the $C^{1}$-functional defined by

$$
\psi_{\lambda}(u)=\frac{1}{p} \vartheta(u)-\frac{\lambda}{p}\|u\|_{p}^{p} \text { for all } u \in W^{1, p}(\Omega) .
$$

We consider the homotopy $h(t, u)$ defined by

$$
h(t, u)=(1-t) \varphi(u)+t \psi_{\lambda}(u) \text { for all } t \in[0,1] \text {, and for all } u \in W^{1, p}(\Omega) .
$$

Claim 3. There exist $k_{0} \in \mathbf{R}$ and $\delta_{0}>0$ such that

$$
h(t, u) \leqslant k_{0} \Rightarrow(1+\|u\|)\left\|h_{u}^{\prime}(t, u)\right\|_{*} \geqslant \delta_{0} \text { for all } t \in[0,1] .
$$

We argue indirectly. So, suppose that Claim 3 is not true. Note that the homotopy $h(t, u)$ maps bounded sets to bounded sets. So, we can find $\left\{t_{n}\right\}_{n \geqslant 1} \subseteq[0,1]$ and $\left\{u_{n}\right\}_{n \geqslant 1} \subseteq W^{1, p}(\Omega)$ such that

(46) $t_{n} \rightarrow t,\left\|u_{n}\right\| \rightarrow \infty, h\left(t_{n}, u_{n}\right) \rightarrow-\infty$ and $\left(1+\left\|u_{n}\right\|\right) h_{u}^{\prime}\left(t_{n}, u_{n}\right) \rightarrow 0$ in $W^{1, p}(\Omega)^{*}$.

From the last convergence in (46), we have

$$
\begin{aligned}
& \left.\left|\left\langle A\left(u_{n}\right), h\right\rangle+\int_{\Omega} \xi(z)\right| u_{n}\right|^{p-2} u_{n} h d z+\int_{\partial \Omega} \beta(z)\left|u_{n}\right|^{p-2} u_{n} h d \sigma \\
& -\left(1-t_{n}\right) \int_{\Omega} f\left(z, u_{n}\right) h d z-t_{n} \lambda \int_{\Omega}\left|u_{n}\right|^{p-2} u_{n} h d z \mid \leqslant \frac{\epsilon_{n}\|h\|}{1+\left\|u_{n}\right\|}
\end{aligned}
$$

for all $h \in W^{1, p}(\Omega)$, with $\epsilon_{n} \rightarrow 0^{+}$.

Let $y_{n}=\frac{u_{n}}{\left\|u_{n}\right\|}, n \in \mathbf{N}$. Then $\left\|y_{n}\right\|=1$ for all $n \in \mathbf{N}$ and so we may assume that

$$
y_{n} \stackrel{w}{\rightarrow} y \text { in } W^{1, p}(\Omega) \text { and } y_{n} \rightarrow y \text { in } L^{p}(\Omega) \text { and } L^{p}(\partial \Omega) .
$$

From (47) we have

$$
\begin{aligned}
& \left.\left|\left\langle A\left(y_{n}\right), h\right\rangle+\int_{\Omega} \xi(z)\right| y_{n}\right|^{p-2} y_{n} h d z+\int_{\partial \Omega} \beta(z)\left|y_{n}\right|^{p-2} y_{n} h d \sigma \\
& -\left(1-t_{n}\right) \int_{\Omega} \frac{f\left(z, u_{n}\right)}{\left\|u_{n}\right\|^{p-1}} h d z-t_{n} \lambda \int_{\Omega}\left|y_{n}\right|^{p-2} y_{n} h d z \mid \leqslant \frac{\epsilon_{n}\|h\|}{1+\left\|u_{n}\right\|} \text { for all } n \in \mathbf{N} .
\end{aligned}
$$


Recall that

$$
\left\{\frac{f\left(\cdot, u_{n}(\cdot)\right)}{\left\|u_{n}\right\|^{p-1}}\right\}_{n \geqslant 1} \subseteq L^{p^{\prime}}(\Omega) \text { is bounded (see hypotheses } H_{1}(\mathrm{i}),(\mathrm{ii}) \text { ). }
$$

Hypothesis $H_{1}(\mathrm{ii})$ and (50) imply that at least for a subsequence, we have

$$
\frac{f\left(\cdot, u_{n}(\cdot)\right)}{\left\|u_{n}\right\|^{p-1}} \stackrel{w}{\rightarrow} \hat{\lambda}_{m}|y|^{p-2} y \text { in } L^{p^{\prime}}(\Omega)
$$

(see Aizicovici, Papageorgiou and Staicu [1], proof of Proposition 30).

In (49) we choose $h=y_{n}-y \in W^{1, p}(\Omega)$, pass to the limit as $n \rightarrow \infty$ and use (48), (51). Then

$$
\begin{aligned}
& \lim _{n \rightarrow \infty}\left\langle A\left(y_{n}\right), y_{n}-y\right\rangle=0, \\
& \Rightarrow y_{n} \rightarrow y \text { in } W^{1, p}(\Omega) \quad \text { (see Proposition 6), hence }\|y\|=1 .
\end{aligned}
$$

So, if in (49) we pass to the limit as $n \rightarrow \infty$ and use (51) and (52), then

$$
\langle A(y), h\rangle+\int_{\Omega} \xi(z)|y|^{p-2} y h d z+\int_{\partial \Omega} \beta(z)|y|^{p-2} y h d \sigma=\lambda_{t} \int_{\Omega}|y|^{p-2} y h d z
$$

for all $h \in W^{1, p}(\Omega)$ with $\lambda_{t}=(1-t) \hat{\lambda}_{m}+t \lambda$,

$$
\Rightarrow-\Delta_{p} y(z)+\xi(z)|y(z)|^{p-2} y(z)=\lambda_{t}|y(z)|^{p-2} y(z) \text { for almost all } z \in \Omega \text {, }
$$

$$
\frac{\partial y}{\partial n_{p}}+\beta(z)|y|^{p-2} y=0 \text { on } \partial \Omega \quad \text { (see Papageorgiou and Rădulescu [18]). }
$$

If $\lambda_{t} \notin \sigma_{0}(p)$, then it follows from (53) that

$$
y=0, \text { a contradiction (see }(52)) \text {. }
$$

So, suppose that $\lambda_{t} \in \sigma_{0}(p)$. If $D=\{z \in \Omega: y(z) \neq 0\}$, then from (52) we see that

$$
|D|_{N}>0 \text { and }\left|u_{n}(z)\right| \rightarrow \infty \text { for almost all } z \in D \text {. }
$$

Reasoning as in the proof of Proposition 10, we show that

$$
\int_{\Omega}\left[f\left(z, u_{n}\right) u_{n}-p F\left(z, u_{n}\right)\right] d z \rightarrow+\infty \text { as } n \rightarrow \infty .
$$

From the third convergence in (46), we have

$$
\vartheta\left(u_{n}\right)-\left(1-t_{n}\right) \int_{\Omega} p F\left(z, u_{n}\right) d z-t_{n} \lambda\left\|u_{n}\right\|_{p}^{p} \leqslant-1 \text { for all } n \geqslant n_{0} .
$$

In (47) we choose $h=u_{n} \in W^{1, p}(\Omega)$. Then

$$
-\vartheta\left(u_{n}\right)+\left(1-t_{n}\right) \int_{\Omega} f\left(z, u_{n}\right) u_{n} d z+t_{n} \lambda\left\|u_{n}\right\|_{p}^{p} \leqslant \epsilon_{n} \text { for all } n \in \mathbf{N}
$$

By choosing $n_{0} \in \mathbf{N}$ even bigger if necessary, we can have

$$
\epsilon_{n} \in(0,1) \text { for all } n \geqslant n_{0} .
$$

Adding (55) and (56), we obtain

$$
\left(1-t_{n}\right) \int_{\Omega}\left[f\left(z, u_{n}\right) u_{n}-p F\left(z, u_{n}\right)\right] d z \leqslant 0 \text { for all } n \geqslant n_{0} .
$$

We may assume that $t_{n} \in[0,1)$ for all $n \geqslant n_{0}$. Otherwise, there exists a subsequence $\left\{t_{n_{k}}\right\}_{k \geqslant 1}$ of $\left\{t_{n}\right\}_{n \geqslant 1}$ with $t_{n_{k}}=1$ for all $k \in \mathbf{N}$. Hence $t=1$ and so 
$\lambda_{t}=\lambda \notin \sigma_{0}(p)$, a contradiction (recall that we have assumed that $\lambda_{t} \in \sigma_{0}(p)$ ). Therefore $t_{n} \in[0,1)$ for all $n \geqslant n_{0}$ and so from $(57)$, we have

$$
\int_{\Omega}\left[f\left(z, u_{n}\right) u_{n}-p F\left(z, u_{n}\right)\right] d z \leqslant 0 \text { for all } n \geqslant n_{0} .
$$

Comparing (54) and (58), we have a contradiction. This proves Claim 3.

Note that the above argument also shows that for every $t \in[0,1], h(t, \cdot)$ satisfies the C-condition. We apply Theorem 5.1.21 of Chang [5, p. 334] (see also Liang and $\mathrm{Su}[13$, Proposition 3.2]) and obtain

$$
\begin{aligned}
& C_{k}(h(0, \cdot), \infty)=C_{k}(h(1, \cdot), \infty) \text { for all } k \in \mathbf{N}_{0}, \\
& \Rightarrow C_{k}(\varphi, \infty)=C_{k}\left(\psi_{\lambda}, \infty\right) \text { for all } k \in \mathbf{N}_{0} .
\end{aligned}
$$

Since $\lambda \notin \sigma_{0}(p)$, we have

$$
\begin{aligned}
& K_{\psi_{\lambda}}=\{0\}, \\
& \Rightarrow C_{k}\left(\psi_{\lambda}, \infty\right)=C_{k}\left(\psi_{\lambda}, 0\right) \text { for all } k \in \mathbf{N}_{0}, \\
& \Rightarrow C_{0}\left(\psi_{\lambda}, \infty\right)=C_{1}\left(\psi_{\lambda}, \infty\right)=0 \quad(\text { see Proposition } 8), \\
& \Rightarrow C_{0}(\varphi, \infty)=C_{1}(\varphi, \infty)=0 \quad(\text { see }(59))
\end{aligned}
$$

Next, we show that $C_{m}(\varphi, \infty) \neq 0$. We introduce the following two sets

$$
\begin{aligned}
G_{r} & =\left\{u \in W^{1, p}(\Omega): \vartheta(u)<\lambda\|u\|_{p}^{p},\|u\|=r\right\} \quad(r>0), \\
H & =\left\{u \in W^{1, p}(\Omega): \vartheta(u) \geqslant \lambda\|u\|_{p}^{p}\right\} .
\end{aligned}
$$

They are symmetric sets and $G_{r} \cap H=\emptyset$. Also let

$$
\partial B_{r}=\left\{u \in W^{1, p}(\Omega):\|u\|=r\right\} .
$$

This is a Banach $C^{1}$-manifold and hence locally contractible. The set $G_{r} \subseteq \partial B_{r}$ is open and thus locally contractible, too. The set $W^{1, p}(\Omega) \backslash H$ is open and of course, locally contractible. By ind $(\cdot)$ we denote the Fadell and Rabinowitz [8] cohomological index. Since $\lambda \in\left(\hat{\lambda}_{m}, \hat{\lambda}_{m+1}\right) \backslash \sigma_{0}(p)$, we have

$$
\text { ind } G_{r}=\operatorname{ind}\left(W^{1, p}(\Omega) \backslash H\right)=m \text {. }
$$

Theorem 3.6 of Cingolani and Degiovanni [4] implies that we can find $K \subseteq$ $W^{1, p}(\Omega)$ compact such that $\left(G_{r} \cup K, G_{r}\right)$ homologically links $H$ in dimension $m \geqslant 2$ (see Motreanu, Motreanu and Papageorgiou [15, p. 167]). So, Theorem 3.2 of [4] says that

$$
\begin{aligned}
& C_{m}\left(\psi_{\lambda}, 0\right) \neq 0, \\
& \Rightarrow C_{m}\left(\psi_{\lambda}, \infty\right) \neq 0 \quad(\text { see }(60)), \\
& \Rightarrow C_{m}(\varphi, \infty) \neq 0 \quad(\text { see }(59)) .
\end{aligned}
$$

Now, we are ready to prove our first existence theorem.

Theorem 13. If hypotheses $H(\xi), H(\beta), H_{1}$ hold, then problem (1) admits a nontrivial solution $\hat{u} \in C^{1}(\bar{\Omega})$.

Proof. From Propositions 10 and 11 we have

$$
C_{1}(\varphi, 0) \neq 0 \quad \text { and } \quad C_{1}(\varphi, \infty)=0 .
$$


So, Corollary 6.92 of Motreanu, Motreanu and Papageorgiou [15, p. 173], implies that we can find $u_{0} \in K_{\varphi}$ such that

$$
\begin{aligned}
\varphi\left(u_{0}\right)<\varphi(0) & =0 \text { and } C_{0}\left(\varphi, u_{0}\right) \neq 0 \\
\text { or } \quad \varphi\left(u_{0}\right)>\varphi(0) & =0 \text { and } C_{2}\left(\varphi, u_{0}\right) \neq 0 .
\end{aligned}
$$

Evidently, in both cases $u_{0} \neq 0$ and it solves problem (1). Moreover, from Papageorgiou and Rădulescu [20] we have $u_{0} \in L^{\infty}(\Omega)$ and hence Theorem 2 of Lieberman [14] implies that $u_{0} \in C^{1}(\bar{\Omega})$.

We can obtain another existence theorem if we change the geometry of the problem near the origin. In this case we allow resonance at $+\infty$ with any variational eigenvalue.

So, the new hypotheses on the reaction $f(z, x)$ are the following:

$H_{2}: f: \Omega \times \mathbf{R} \rightarrow \mathbf{R}$ is a Carathéodory function such that $f(z, 0)=0$ for almost all $z \in \Omega$ and

(i) for every $\rho>0$, there exists $a_{\rho} \in L^{\infty}(\Omega)$, such that

$$
|f(z, x)| \leqslant a_{\rho}(z) \text { for almost all } z \in \Omega \text {, and for all }|x| \leqslant \rho ;
$$

(ii) there exists $m \in \mathbf{N}$ such that

$$
\lim _{x \rightarrow \pm \infty} \frac{f(z, x)}{|x|^{p-2} x}=\hat{\lambda}_{m} \text { uniformly for almost all } z \in \Omega
$$

(iii) if $F(z, x)=\int_{0}^{x} f(z, s) d s$, then $\lim _{x \rightarrow \pm \infty}[f(z, x) x-p F(z, x)]=+\infty$ uniformly for almost all $z \in \Omega$;

(iv) there exists $\eta \in L^{\infty}(\Omega), \eta(z) \leqslant \hat{\lambda}_{1}$ for almost all $z \in \Omega, \eta \not \equiv \hat{\lambda}_{1}$ such that

$$
\limsup _{x \rightarrow 0} \frac{p F(z, x)}{|x|^{p}} \leqslant \eta(z) \text { uniformly for almost all } z \in \Omega \text {. }
$$

Lemma 14. If $\eta \in L^{\infty}(\Omega), \eta(z) \leqslant \hat{\lambda}_{1}$ for almost all $z \in \Omega, \eta \not \hat{\lambda}_{1}$, then there exists $c_{6}>0$ such that

$$
\psi(u)=\vartheta(u)-\int_{\Omega} \eta(z)|u|^{p} d z \geqslant c_{6}\|u\|^{p} \text { for all } u \in W^{1, p}(\Omega) .
$$

Proof. From (3) we see that $\psi \geqslant 0$. Arguing indirectly, suppose that the lemma is not true. Exploiting the $p$-homogeneity of $\psi(\cdot)$ we can find $\left\{u_{n}\right\}_{n \geqslant 1} \subseteq W^{1, p}(\Omega)$ such that

$$
\left\|u_{n}\right\|=1 \text { for all } n \in \mathbf{N} \text { and } \psi\left(u_{n}\right) \downarrow 0 .
$$

We may assume that

$$
u_{n} \stackrel{w}{\rightarrow} u \text { in } W^{1, p}(\Omega) \text { and } u_{n} \rightarrow u \text { in } L^{p}(\Omega) \text { and in } L^{p}(\partial \Omega) .
$$

Then in the limit as $n \rightarrow \infty$, we have

$$
\begin{aligned}
& \psi(u) \leqslant 0 \quad(\text { see }(6)), \\
& \Rightarrow \vartheta(u) \leqslant \int_{\Omega} \eta(z)|u|^{p} d z \leqslant \hat{\lambda}_{1}\|u\|_{p}^{p} \\
& \Rightarrow \vartheta(u)=\hat{\lambda}_{1}\|u\|_{p}^{p} \quad(\text { see }(3)), \\
& \Rightarrow u=k \hat{u}_{1} \quad \text { with } k \in \mathbf{R} .
\end{aligned}
$$

If $k=0$, then $u=0$ and we have $u_{n} \rightarrow 0$ in $W^{1, p}(\Omega)$, a contradiction (see (61)). 
If $k \neq 0$, then $|u(z)| \neq 0$ for all $z \in \bar{\Omega}$ (recall that $\hat{u}_{1} \in D_{+}$). From (62) and the hypothesis on $\eta(\cdot)$, we infer that

$$
\vartheta(u)<\hat{\lambda}_{1}\|u\|_{p}^{p}
$$

which contradicts (3). Therefore the lemma is true.

We can now prove our second existence theorem.

Theorem 15. If hypotheses $H(\xi), H(\beta), H_{2}$ hold, then problem (1) admits a nontrivial solution $u_{0} \in C^{1}(\bar{\Omega})$.

Proof. Hypothesis $H_{2}(i v)$ implies that given $\epsilon>0$, we can find $\delta=\delta(\epsilon)>0$ such that

$$
F(z, x) \leqslant \frac{1}{p}(\eta(z)+\epsilon)|x|^{p} \text { for almost all } z \in \Omega, \text { and for all }|x| \leqslant \delta
$$

Let $u \in C^{1}(\bar{\Omega})$ with $\|u\|_{C^{1}(\bar{\Omega})} \leqslant \delta$. We have

$$
\begin{aligned}
\varphi(u) & =\frac{1}{p} \vartheta(u)-\int_{\Omega} F(z, u) d z \\
& \geqslant \frac{1}{p}\left[\vartheta(u)-\int_{\Omega} \eta(z)|u|^{p} d z\right]-\epsilon\|u\|^{p} \text { (see (63)) } \\
& \geqslant\left(c_{7}-\epsilon\right)\|u\|^{p} \text { for some } c_{7}>0 \quad \text { (see Lemma 14). }
\end{aligned}
$$

Choosing $\epsilon \in\left(0, c_{7}\right)$ we infer that

$$
\begin{aligned}
& \varphi(u) \geqslant 0 \text { for all } u \in C^{1}(\bar{\Omega}),\|u\|_{C^{1}(\bar{\Omega})} \leqslant \delta \\
& \Rightarrow u=0 \text { is a local } C^{1}(\bar{\Omega}) \text {-minimizer of } \varphi \\
& \left.\Rightarrow u=0 \text { is a local } W^{1, p}(\Omega) \text {-minimizer of } \varphi \quad \text { (see Proposition } 5\right), \\
& \Rightarrow C_{k}(\varphi, 0)=\delta_{k, 0} \mathbf{Z} \text { for all } k \in \mathbf{N}_{0} .
\end{aligned}
$$

On the other hand, from Proposition 12 we have $C_{m}(\varphi, \infty) \neq 0$. So, from Theorem 6.62 of Motreanu, Motreanu and Papageorgiou [15, p. 160], we know that we can find $u_{0} \in K_{\varphi}$ such that

$$
C_{m}\left(\varphi, u_{0}\right) \neq 0, \quad m \geqslant 1
$$

Comparing (64) and (65), we infer that $u_{0} \neq 0$. As before, the nonlinear regularity theory implies that $u_{0} \in C^{1}(\bar{\Omega})$.

\section{Resonance with respect to the principal eigenvalue}

In this section, we examine problems which are resonant with respect to the principal eigenvalue. The problem under consideration is the following:

$$
\begin{cases}-\Delta_{p} u(z)+\xi(z)|u(z)|^{p-2} u(z)=\hat{\lambda}_{1}|u(z)|^{p-2} u(z)+g(z, u(z)) & \text { in } \Omega \\ \frac{\partial u}{\partial n_{p}}+\beta(z)|u|^{p-2} u=0 & \text { on } \partial \Omega\end{cases}
$$

The hypotheses on the perturbation $g(z, x)$ are the following:

$H_{3}: g: \Omega \times \mathbf{R} \rightarrow \mathbf{R}$ is a Carathéodory function such that $g(z, 0)=0$ for almost all $z \in \Omega$ and 
(i) if $G(z, x)=\int_{0}^{x} g(z, s) d s$, then there exist functions $G_{ \pm} \in L^{1}(\Omega)$ such that

$$
\int_{\Omega} G_{ \pm}(z) d z \leqslant 0, \quad g(z, x) \rightarrow 0 \text { and } G(z, x) \rightarrow G_{ \pm}(z)
$$

uniformly for almost all $z \in \Omega$, as $x \rightarrow \pm \infty$;

and for every $\rho>0$ there exists $a_{\rho} \in L^{\infty}(\Omega)_{+}$such that

$$
|g(z, x)| \leqslant a_{\rho}(z) \text { for almost all } z \in \Omega \text {, and for all }|x| \leqslant \rho \text {. }
$$

(ii) $G(z, x) \leqslant \frac{1}{p}\left[\hat{\lambda}(p)-\hat{\lambda}_{1}\right]|x|^{p}$ for almost all $z \in \Omega$, and for all $x \in \mathbf{R}$;

(iii) there exists a function $\eta \in L^{\infty}(\Omega)$ such that

$$
\begin{aligned}
& \eta(z) \geqslant 0 \text { for almost all } z \in \Omega, \eta \neq 0, \\
& \liminf _{x \rightarrow 0} \frac{p G(z, x)}{|x|^{p}} \geqslant \eta(z) \text { uniformly for almost all } z \in \Omega .
\end{aligned}
$$

Remark 4. Because of hypothesis $H_{3}(\mathrm{i})$, in the terminology introduced by Landesman and Lazer [12], the problem is "strongly resonant" with respect to the principal eigenvalue. Such problems exhibit a partial lack of compactness (that is, the energy (Euler) functional of the problem does not satisfy the C-condition at all levels). This is evident from Proposition 16 below.

The energy functional $\varphi: W^{1, p}(\Omega) \rightarrow \mathbf{R}$ is defined by

$$
\varphi(u)=\frac{1}{p} \vartheta(u)-\frac{\hat{\lambda}_{1}}{p}\|u\|_{p}^{p}-\int_{\Omega} G(z, u) d z \text { for all } u \in W^{1, p}(\Omega) .
$$

We have $\varphi \in C^{1}\left(W^{1, p}(\Omega)\right)$.

Proposition 16. If hypotheses $H(\xi), H(\beta), H_{3}$ hold, then the functional $\varphi$ satisfies the $C_{c}$-condition for every

$$
c<\min \left\{-\int_{\Omega} G_{+}(z) d z,-\int_{\Omega} G_{-}(z) d z\right\} .
$$

Proof. Let $m_{0}=\min \left\{-\int_{\Omega} G_{+}(z) d z,-\int_{\Omega} G_{-}(z) d z\right\}$ and let $c<m_{0}$. We consider a sequence such that

$$
\begin{aligned}
& \varphi\left(u_{n}\right) \rightarrow c, \\
& \left(1+\left\|u_{n}\right\|\right) \varphi^{\prime}\left(u_{n}\right) \rightarrow 0 \text { in } W^{1, p}(\Omega)^{*} .
\end{aligned}
$$

Claim 4. $\left\{u_{n}\right\}_{n \geqslant 1} \subseteq W^{1, p}(\Omega)$ is bounded.

Arguing by contradiction, suppose that the claim is not true. By passing to a subsequence if necessary, we may assume that

$$
\left\|u_{n}\right\| \rightarrow \infty
$$

Let $y_{n}=\frac{u_{n}}{\left\|u_{n}\right\|}, n \in \mathbf{N}$. Then $\left\|y_{n}\right\|=1$ for all $n \in \mathbf{N}$ and so we may assume that

$$
y_{n} \stackrel{w}{\rightarrow} y \text { in } W^{1, p}(\Omega) \text { and } y_{n} \rightarrow y \text { in } L^{p}(\Omega) \text { and in } L^{p}(\partial \Omega) .
$$


From (67) we have

$$
\begin{aligned}
& \frac{1}{p} \vartheta\left(u_{n}\right)-\frac{\hat{\lambda}_{1}}{p}\left\|u_{n}\right\|_{p}^{p}-\int_{\Omega} G\left(z, u_{n}\right) d z \leqslant M_{3} \text { for some } M_{3}>0, \text { and for all } n \in \mathbf{N}, \\
& \Rightarrow \frac{1}{p} \vartheta\left(y_{n}\right)-\frac{\hat{\lambda}_{1}}{p}\left\|y_{n}\right\|_{p}^{p}-\int_{\Omega} \frac{G\left(z, u_{n}\right)}{\left\|u_{n}\right\|^{p}} d z \leqslant \frac{M_{3}}{\left\|u_{n}\right\|^{p}} \text { for all } n \in \mathbf{N}, \\
& \Rightarrow \vartheta(y) \leqslant \hat{\lambda}_{1}\|y\|_{p}^{p} \quad\left(\text { see }(69),(70) \text { and hypothesis } H_{3}(i)\right), \\
& \Rightarrow \vartheta(y)=\hat{\lambda}_{1}\|y\|_{p}^{p} \quad(\text { see }(3)), \\
& \Rightarrow y=k \hat{u}_{1} \text { with } k \in \mathbf{R} .
\end{aligned}
$$

If $k=0$, then $y_{n} \rightarrow 0$ in $W^{1, p}(\Omega)$, a contradiction to the fact that $\left\|y_{n}\right\|=1$ for all $n \in \mathbf{N}$.

If $k \neq 0$, then to fix things we assume that $k>0$ (the reasoning is similar if $k<0)$. We have

$$
u_{n}(z) \rightarrow+\infty \text { for almost all } z \in \Omega \text {, as } n \rightarrow \infty \text {. }
$$

From (67) we see that given $\epsilon>0$, we can find $n_{0}=n_{0}(\epsilon) \in \mathbf{N}$ such that

$$
\begin{aligned}
& \varphi\left(u_{n}\right) \leqslant c+\epsilon \text { for all } n \geqslant n_{0}, \\
& \Rightarrow \frac{1}{p} \vartheta\left(u_{n}\right)-\frac{\hat{\lambda}_{1}}{p}\left\|u_{n}\right\|_{p}^{p}-\int_{\Omega} G\left(z, u_{n}\right) d z \leqslant c+\epsilon \text { for all } n \geqslant n_{0} .
\end{aligned}
$$

From (3) we have

$$
\hat{\lambda}_{1}\left\|u_{n}\right\|_{p}^{p} \leqslant \vartheta\left(u_{n}\right) \text { for all } n \in \mathbf{N} .
$$

Using this in (72), we obtain

$$
\begin{aligned}
& -\int_{\Omega} G\left(z, u_{n}\right) d z \leqslant c+\epsilon \text { for all } n \geqslant n_{0}, \\
& \Rightarrow-\int_{\Omega} G_{+}(z) d z \leqslant c+\epsilon \text { (from }(71) \text {, hypothesis } H_{3}(i) \text { and Fatou's lemma). }
\end{aligned}
$$

Since $\epsilon>0$ is arbitrary, we let $\epsilon \rightarrow 0^{+}$and conclude that

$$
-\int_{\Omega} G_{+}(z) d z \leqslant c
$$

which contradicts the choice of $c<m_{0}$. This proves Claim 4 .

Because of Claim 4 we may assume that

$$
u_{n} \stackrel{w}{\rightarrow} u \text { in } W^{1, p}(\Omega) \text { and } u_{n} \rightarrow u \text { in } L^{p}(\Omega) \text { and in } L^{p}(\partial \Omega) .
$$

From (68) we have

$$
\begin{aligned}
& \left.\left|\left\langle A\left(u_{n}\right), h\right\rangle+\int_{\Omega} \xi(z)\right| u_{n}\right|^{p-2} u_{n} h d z+\int_{\partial \Omega} \beta(z)\left|u_{n}\right|^{p-2} u_{n} h d \sigma \\
& -\hat{\lambda}_{1} \int_{\Omega}\left|u_{n}\right|^{p-2} u_{n} h d z-\int_{\Omega} g\left(z, u_{n}\right) h d z \mid \leqslant \frac{\epsilon_{n}\|h\|}{1+\left\|u_{n}\right\|}
\end{aligned}
$$

for all $h \in W^{1, p}(\Omega)$ with $\epsilon_{n} \rightarrow 0^{+}$. 
In (74) we choose $h=u_{n}-u \in W^{1, p}(\Omega)$, pass to the limit as $n \rightarrow \infty$ and use (73). Then

$$
\begin{aligned}
& \lim _{n \rightarrow \infty}\left\langle A\left(u_{n}\right), u_{n}-u\right\rangle=0, \\
& \Rightarrow u_{n} \rightarrow u \text { in } W^{1, p}(\Omega) \quad(\text { see Proposition } 6) \\
& \Rightarrow \varphi \text { satisfies the } C_{c^{-}} \text {condition for } c<m_{0} .
\end{aligned}
$$

The proof is complete.

Now we prove a multiplicity theorem for problem (66), producing two nontrivial smooth solutions.

Theorem 17. If hypotheses $H(\xi), H(\beta), H_{3}$ hold, then problem (66) admits at least two nontrivial solutions

$$
u_{0}, \hat{u} \in C^{1}(\bar{\Omega}) .
$$

Proof. Hypothesis $H_{3}$ (iii) implies that given $\epsilon>0$, we can find $\delta=\delta(\epsilon)>0$ such that

$$
G(z, x) \geqslant \frac{1}{p}(\eta(z)-\epsilon)|x|^{p} \text { for almost all } z \in \Omega, \text { and for all }|x| \leqslant \delta .
$$

Recall that $\hat{u}_{1} \in D_{+}$. So, for small $t \in(0,1)$ we will have

$$
t \hat{u}_{1}(z) \in(0, \delta] \text { for all } z \in \bar{\Omega} \text {. }
$$

Then we have

$$
\begin{aligned}
\varphi\left(t \hat{u}_{1}\right) & =\frac{t^{p}}{p}\left[\vartheta\left(\hat{u}_{1}\right)-\hat{\lambda}_{1}\right]-\int_{\Omega} G\left(z, t \hat{u}_{1}\right) d z \quad\left(\text { recall that }\left\|\hat{u}_{1}\right\|_{p}=1\right) \\
& =-\int_{\Omega} G\left(z, t \hat{u}_{1}\right) d z \\
& \leqslant \frac{t^{p}}{p}\left[\epsilon-\int_{\Omega} \eta(z) \hat{u}_{1}^{p} d z\right] \quad\left(\text { see }(75),(76) \text { and recall that }\left\|\hat{u}_{1}\right\|_{p}=1\right) .
\end{aligned}
$$

Since $\hat{u}_{1} \in D_{+}$and $\eta \not \equiv 0$ (see hypothesis $H_{3}($ iii) $)$, we have

$$
0<\tau_{0}=\int_{\Omega} \eta(z) \hat{u}_{1}^{p} d z
$$

Then from (77) and by choosing $\epsilon \in\left(0, \tau_{0}\right)$, we obtain

$$
\varphi\left(t \hat{u}_{1}\right)<0 \text {. }
$$

Because $\vartheta(u) \geqslant \hat{\lambda}_{1}\|u\|_{p}^{p}$ for all $u \in W^{1, p}(\Omega)$ (see (3)) and using hypothesis $H_{3}(i)$ we infer that

$$
\varphi \text { is bounded below. }
$$

So, in conjunction with (78) we have

$$
-\infty<m=\inf \varphi<0=\varphi(0) .
$$
that

From hypothesis $H_{3}(\mathrm{i})$ we see that $m_{0} \geqslant 0$. Therefore Proposition 16 implies $\varphi$ satisfies the $C_{m}$-condition.

Invoking Proposition 1 , we can find $u_{0} \in W^{1, p}(\Omega)$ such that

$$
\begin{aligned}
& m=\varphi\left(u_{0}\right)<0=\varphi(0) \quad(\text { see }(79)), \\
& \Rightarrow u_{0} \neq 0 .
\end{aligned}
$$


Since $u_{0} \in K_{\varphi}$, it follows that $u_{0}$ is a nontrivial solution of (66) and as before, the nonlinear regularity theory implies that

$$
u_{0} \in C^{1}(\bar{\Omega})
$$

Next, we consider the following direct sum decomposition

$$
W^{1, p}(\Omega)=\mathbf{R} \hat{u}_{1} \oplus V_{p}
$$

If $u \in V_{p}$, then

$$
\begin{aligned}
\varphi(u) & \left.\geqslant \frac{1}{p} \vartheta(u)-\frac{\hat{\lambda}_{1}}{p}\|u\|_{p}^{p}-\frac{1}{p}\left[\hat{\lambda}(p)-\hat{\lambda_{1}}\right]\|u\|_{p}^{p} \quad \text { (see hypothesis } H_{3}(\mathrm{ii})\right) \\
& =\frac{1}{p} \vartheta(u)-\frac{\hat{\lambda}(p)}{p}\|u\|_{p}^{p} \\
& \geqslant 0 \quad(\text { see }(6)) \\
& \Rightarrow \inf _{V_{p}} \varphi \geqslant 0 .
\end{aligned}
$$

On the other hand, if $r \in(0,1)$ is small, then from $(78)$ and $(76)$ we see that

$$
\mu=\sup \left[\varphi(u): u \in \bar{B}_{r} \cap \mathbf{R} \hat{u}_{1}\right]<0
$$

with $\bar{B}_{r}=\left\{u \in W^{1, p}(\Omega):\|u\| \leqslant r\right\}$. We consider the following family of maps

$$
\Gamma=\left\{\gamma \in C\left(\bar{B}_{r} \cap \mathbf{R} \hat{u}_{1}, W^{1, p}(\Omega)\right):\left.\gamma\right|_{\partial \bar{B}_{r} \cap \mathbf{R} \hat{u}_{1}}=\left.i d\right|_{\partial \bar{B}_{r} \cap \mathbf{R} \hat{u}_{1}}\right\} .
$$

We assume that

$$
K_{\varphi}=\left\{0, u_{0}\right\}
$$

Otherwise we already have a second nontrivial solution $\hat{u}_{1}$, which by the nonlinear regularity theory belongs to $C^{1}(\bar{\Omega})$ and so we are done.

Let $b=0$ and $a=m=\varphi\left(u_{0}\right)$ and let $h(t, u)$ be the deformation postulated by Theorem 2 (the second deformation theorem). From (83) we see that $K_{\varphi}^{b}=\{0\}$ and $\varphi^{a}=\left\{u_{0}\right\}$. Hence

$$
h(1, u)=u_{0} \text { for all } u \in V_{p}
$$

Also, if $\|u\|=\frac{r}{2}$, then

$$
h\left(\frac{2(r-\|u\|)}{r}, \frac{r u}{\|u\|}\right)=h(1,2 u)=u_{0} \quad(\text { since } 2\|u\|=r, \text { see }(81),(84)) .
$$

So, if we consider the map $\gamma_{0}: \bar{B}_{r} \cap \mathbf{R} \hat{u}_{1} \rightarrow W^{1, p}(\Omega)$ defined by

$$
\gamma_{0}(u)= \begin{cases}u_{0} & \text { if }\|u\|<\frac{r}{2} \\ h\left(\frac{2(r-\|u\|)}{r}, \frac{r u}{\|u\|}\right) & \text { if }\|u\| \geqslant \frac{r}{2}\end{cases}
$$

then from the previous remarks we see that $\gamma_{0}$ is continuous. Also, if $\|u\|=r$ then

$$
\begin{aligned}
& h(0, u)=u, \\
& \left.\Rightarrow \gamma_{0}\right|_{\partial \bar{B}_{r} \cap \mathbf{R} \hat{u}_{1}}=\left.\mathrm{id}\right|_{\partial \bar{B}_{r} \cap \mathbf{R} \hat{u}_{1}}, \\
& \Rightarrow \gamma_{0} \in \Gamma \quad(\text { see }(82)) .
\end{aligned}
$$

From Theorem 2 (the second deformation theorem), we know that the homotopy $h(t, u)$ is $\varphi$-decreasing. Thus, it follows from (81) that

$$
\varphi\left(\gamma_{0}(u)\right)<0 \text { for all } u \in \bar{B}_{r} \cap \mathbf{R} u_{1} .
$$


From Example 5.2.3(b) of Gasinski and Papageorgiou [9, p. 642], we know that the sets $\partial \bar{B}_{r} \cap \mathbf{R} \hat{u}_{1}$ and $V_{p}$ link in $W^{1, p}(\Omega)$ (see Definition 3). Therefore we have

$$
\begin{aligned}
& \gamma\left(\bar{B}_{r} \cap \mathbf{R} \hat{u}_{1}\right) \cap V_{p} \neq \emptyset \text { for all } \gamma \in \Gamma, \\
& \left.\Rightarrow \sup \left[\gamma(u): u \in \bar{B}_{r} \cap \mathbf{R} \hat{u}_{1}\right] \geqslant 0 \text { for all } \gamma \in \Gamma \text { (see }(80)\right), \\
& \Rightarrow \sup \left[\gamma_{0}(u): u \in \bar{B}_{r} \cap \mathbf{R} \hat{u}_{1}\right]=\gamma_{0}(\tilde{u}) \geqslant 0 \text { for some } \tilde{u} \in \bar{B}_{r} \cap \mathbf{R} \hat{u}_{1} .
\end{aligned}
$$

This contradicts (85). So, we can find $\hat{u} \in K_{\varphi}, \hat{u} \notin\left\{0, u_{0}\right\}$. Then $\hat{u}$ is the second nontrivial solution of (66). As before, the nonlinear regularity theory implies that $\hat{u} \in C^{1}(\bar{\Omega})$.

We can have a three solutions theorem, if we change the geometry. So, the new conditions on the perturbation term $g(z, x)$ are the following:

$H_{4}: g: \Omega \times \mathbf{R} \rightarrow \mathbf{R}$ is a Carathéodory function such that $g(z, 0)=0$ for almost all $z \in \Omega$ and

(i) if $G(z, x)=\int_{0}^{x} g(z, s) d s$, then there exist functions $G_{ \pm} \in L^{1}(\Omega)$ and constants $c_{-}<0<c_{+}$such that

$$
0<\int_{\Omega} G_{ \pm}(z) d z \leqslant \int_{\Omega} G\left(z, c_{ \pm} \hat{u}_{1}\right) d z, \quad p G(z, x)-g(z, x) x \rightarrow G_{ \pm}(z)
$$

uniformly for almost all $z \in \Omega$ as $x \rightarrow \pm \infty$

and there exist $a \in L^{\infty}(\Omega)_{+}$and $1<r<p^{*}=\left\{\begin{array}{ll}\frac{N p}{N-p} & \text { if } p<N \\ +\infty & \text { if } p \geqslant N\end{array}\right.$ such that

$|g(z, x)| \leqslant a(z)\left(1+|x|^{r-1}\right)$ for almost all $z \in \Omega$, and for all $x \in \mathbf{R}$;

(ii) $G(z, x) \leqslant \frac{1}{p}\left[\hat{\lambda}(p)-\hat{\lambda}_{1}\right]|x|^{p}$ for almost all $z \in \Omega$, and for all $x \in \mathbf{R}$;

(iii) there exists a function $\eta \in L^{\infty}(\Omega)_{+}$such that

$\eta(z) \leqslant 0$ for almost all $z \in \Omega, \eta \neq 0$,

$\limsup _{x \rightarrow 0} \frac{p G(z, x)}{|x|^{p}} \leqslant \eta(z)$ uniformly for almost all $z \in \Omega$.

Remark 5. Again hypothesis $H_{4}(\mathrm{i})$ incorporates in our framework problems which are strongly resonant with respect to the principal eigenvalue.

Proposition 18. If hypotheses $H(\xi), H(\beta), H_{4}$ hold, then the functional $\varphi$ sat-

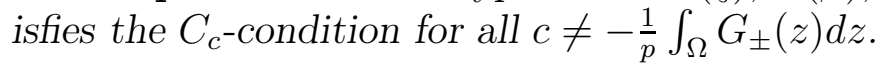

Proof. Consider a Cerami sequence $\left\{u_{n}\right\}_{n \geqslant 1} \subseteq W^{1, p}(\Omega)$ (that is, the sequence satisfies (67) and (68)). We show that $\left\{u_{n}\right\}_{n \geqslant 1} \subseteq W^{1, p}(\Omega)$ is bounded. Arguing by contradiction, assume that $\left\|u_{n}\right\| \rightarrow \infty$ and let $y_{n}=\frac{u_{n}}{\left\|u_{n}\right\|}, n \in \mathbf{N}$. Since $\left\|y_{n}\right\|=1$ for all $n \in \mathbf{N}$, we may assume that

$$
y_{n} \stackrel{w}{\rightarrow} y \text { in } W^{1, p}(\Omega) \text { and } y_{n} \rightarrow y \text { in } L^{p}(\Omega) \text { and in } L^{p}(\partial \Omega) .
$$

Reasoning as in the proof of Proposition 16, we show that $y=k \hat{u}_{1}$, with $k \neq 0$. To fix things, we assume that $k>0$ and so $u_{n}(z) \rightarrow+\infty$ for almost all $z \in \Omega$.

From (67) and (68) we have

$$
\begin{aligned}
& p(c-\epsilon) \leqslant p \varphi\left(u_{n}\right) \leqslant p(c+\epsilon) \text { for all } n \geqslant n_{0}, \\
& \left|\left\langle\varphi^{\prime}\left(u_{n}\right), h\right\rangle\right| \leqslant \frac{\epsilon_{n}\|h\|}{1+\left\|u_{n}\right\|} \text { for all } h \in W^{1, p}(\Omega) \text { with } \epsilon_{n} \rightarrow 0^{+} .
\end{aligned}
$$


Choosing $h=u_{n} \in W^{1, p}(\Omega)$ we obtain

$$
-\epsilon_{n} \leqslant-\left\langle\varphi^{\prime}\left(u_{n}\right), u_{n}\right\rangle \leqslant \epsilon_{n} \text { for all } n \in \mathbf{N} .
$$

Adding (86) and (87), we obtain

$$
p(c-\epsilon)-\epsilon_{n} \leqslant \int_{\Omega}\left[g\left(z, u_{n}\right) u_{n}-p G\left(z, u_{n}\right)\right] d z \leqslant p(c+\epsilon)+\epsilon_{n} \text { for all } n \geqslant n_{0} .
$$

Recalling that $u_{n}(z) \rightarrow+\infty$ for almost all $z \in \Omega$ and that $\epsilon>0$ is arbitrary, if we pass to the limit as $n \rightarrow \infty$ and use hypothesis $H_{4}(i)$, we obtain

$$
c=-\frac{1}{p} \int_{\Omega} G_{+}(z) d z,
$$

a contradiction to our assumption on the level $c$.

We consider the direct sum decomposition

$$
W^{1, p}(\Omega)=\mathbf{R} \hat{u}_{1} \oplus V_{p}
$$

and we introduce the following two open subsets of $W^{1, p}(\Omega)$

$$
U_{+}=\left\{t \hat{u}_{1}+v: t>0, v \in V_{p}\right\} \quad \text { and } \quad U_{-}=\left\{t \hat{u}_{1}+v: t<0, v \in V_{p}\right\} .
$$

Note that

$$
\inf _{\bar{U}_{ \pm}} \varphi \leqslant \varphi\left(c_{ \pm} \hat{u}_{1}\right)=-\int_{\Omega} G\left(z, c_{ \pm} \hat{u}_{1}\right) d z \leqslant-\int_{\Omega} G_{ \pm}(z) d z<0 .
$$

Moreover, as in the proof of Theorem 17, using hypothesis $H_{4}$ (iii), we have that

$$
\inf _{V_{p}} \varphi=0 .
$$

These observations will help us to prove the existence of three nontrivial smooth solutions.

Theorem 19. If hypotheses $H(\xi), H(\beta), H_{4}$ hold, then problem (66) has at least three nontrivial solutions

$$
\hat{u}_{+}, \hat{u}_{-}, \hat{y} \in C^{1}(\bar{\Omega}) .
$$

Proof. Let $\varphi_{+}: W^{1, p}(\Omega) \rightarrow \overline{\mathbf{R}}=\mathbf{R} \cup\{+\infty\}$ be the lower semicontinuous and bounded below functional defined by

$$
\varphi_{+}(u)=\left\{\begin{array}{ll}
\varphi(u) & \text { if } u \in \bar{U}_{+} \\
+\infty & \text { otherwise }
\end{array} \quad \text { (see hypothesis } H_{4}(i)\right) .
$$

Invoking the extended Ekeland variational principle (see, for example, Gasinski and Papageorgiou [9, p. 598]), we can find $\left\{u_{n}\right\}_{n \geqslant 1} \subseteq U_{+}$such that

$$
\begin{aligned}
& \varphi\left(u_{n}\right)=\varphi_{+}\left(u_{n}\right) \downarrow \inf \varphi_{+} \quad\left(\text { recall that } \varphi_{+}\right. \text {is bounded below), } \\
& \varphi\left(u_{n}\right)=\varphi_{+}\left(u_{n}\right) \leqslant \varphi_{+}(y)+\frac{1}{n\left(1+\left\|u_{n}\right\|\right)}\left\|y-u_{n}\right\| \text { for all } y \in W^{1, p}(\Omega) .
\end{aligned}
$$

Since $u_{n} \in U_{+}$for $h \in W^{1, p}(\Omega)$ and $\lambda \in(0,1)$ is small, we have

$$
u_{n}+\lambda h \in U_{+} .
$$


Because $\left.\varphi_{+}\right|_{U_{+}}=\left.\varphi\right|_{U_{+}}$, it follows from (91) with $y=u_{n}+\lambda h$, that

$$
\begin{aligned}
& -\frac{\|h\|}{n\left(1+\left\|u_{n}\right\|\right)} \leqslant \frac{\varphi\left(u_{n}+\lambda h\right)-\varphi\left(u_{n}\right)}{\lambda}, \\
& \Rightarrow-\frac{\|h\|}{n\left(1+\left\|u_{n}\right\|\right)} \leqslant\left\langle\varphi^{\prime}\left(u_{n}\right), h\right\rangle .
\end{aligned}
$$

From Lemma 5.1.38 of Gasinski and Papageorgiou [9, p. 639], we know that we can find $u_{n}^{*} \in W^{1, p}(\Omega)^{*}$ with $\left\|u_{n}^{*}\right\|_{*} \leqslant 1$ such that

$$
\begin{aligned}
& \left\langle u_{n}^{*}, h\right\rangle \leqslant n\left(1+\left\|u_{n}\right\|\right)\left\langle\varphi^{\prime}\left(u_{n}\right), h\right\rangle \text { for all } h \in W^{1, p}(\Omega) \\
& \Rightarrow\left(1+\left\|u_{n}\right\|\right) \varphi^{\prime}\left(u_{n}\right) \rightarrow 0 \in W^{1, p}(\Omega)^{*} \\
& \Rightarrow u_{n} \rightarrow \hat{u}_{+} \in W^{1, p}(\Omega) \quad(\text { see }(86) \text { and Theorem } 17), \\
& \Rightarrow \varphi\left(\hat{u}_{+}\right)=\inf _{\bar{U}_{+}} \varphi=\inf \varphi_{+}<0 \quad(\text { see }(88)) .
\end{aligned}
$$

If $\hat{u}_{+} \in \partial U_{+}$, then $\hat{u}_{+} \in V_{p}$ and so

$$
\varphi\left(\hat{u}_{+}\right) \geqslant 0 \quad(\text { see }(89)) .
$$

This contradicts (92). Therefore $\hat{u}_{+} \in U_{+}$and this means that $\hat{u}_{+}$is a local minimizer of $\varphi$. Moreover, the nonlinear regularity theory implies that $\hat{u}_{+} \in C^{1}(\bar{\Omega})$.

Similarly, using the lower semicontinuous and bounded below functional

$$
\varphi_{-}(u)= \begin{cases}\varphi(u) & \text { if } u \in \bar{U}_{-}, \\ +\infty & \text { otherwise }\end{cases}
$$

we produce $\hat{u}_{-} \in C^{1}(\bar{\Omega})$ a second nontrivial smooth solution of $(66)$, which is also a local minimizer of $\varphi$.

Without any loss of generality we may assume that

$$
\varphi\left(\hat{u}_{-}\right) \leqslant \varphi\left(\hat{u}_{+}\right)
$$

(the reasoning is similar if the opposite inequality holds). We assume that $K_{\varphi}$ is finite (otherwise we already have an infinity of nontrivial solutions of (66) all of them in $C^{1}(\bar{\Omega})$ by the nonlinear regularity theory). Since $\hat{u}_{+}$is a local minimizer of $\varphi$, we can find $\rho \in(0,1)$ small such that

$$
\varphi\left(\hat{u}_{-}\right) \leqslant \varphi\left(\hat{u}_{+}\right)<\inf \left[\varphi(u):\left\|u-\hat{u}_{+}\right\|=\rho\right]=m_{\rho}^{+}\left\|\hat{u}_{-}-\hat{u}_{+}\right\|>\rho
$$

(see [1], proof of Proposition 29).

Let $\Gamma=\left\{\gamma \in C\left([0,1], W^{1, p}(\Omega)\right): \gamma(0)=\hat{u}_{-}, \gamma(1)=\hat{u}_{+}\right\}$and define

$$
c=\inf _{\gamma \in \Gamma} \max _{0 \leqslant t \leqslant 1} \varphi(\gamma(t)) .
$$

Since $\hat{u}_{+} \in U_{+}$and $\hat{u}_{-} \in U_{-}$, we see from (89) that

$$
\begin{aligned}
& c \geqslant 0 \\
& \Rightarrow \varphi \text { satisfies the } C_{c} \text {-condition } \\
& \text { (see Theorem } 17 \text { and hypothesis } H_{1}(\mathrm{i}) \text { ). }
\end{aligned}
$$

Then (93) and (94) permit the use of Theorem 4 (the mountain pass theorem). So, we can find $\hat{y} \in W^{1, p}(\Omega)$ such that

$$
\begin{aligned}
& \hat{y} \in K_{\varphi} \text { and } m_{\rho}^{+} \leqslant \varphi(\hat{y}) \\
& \Rightarrow \hat{y} \in C^{1}(\bar{\Omega}) \text { is a solution of }(66) \text { and } \hat{y} \notin\left\{\hat{u}_{+}, \hat{u}_{-}\right\} \quad \text { (see (93)). }
\end{aligned}
$$


Since $\hat{y}$ is a critical point of $\varphi$ of mountain pass type, we have

$$
C_{1}(\varphi, \hat{y}) \neq 0
$$

(see Motreanu, Motreanu and Papageorgiou [15, p. 168]).

On the other hand, from hypothesis $H_{4}$ (iii) we see that given $\epsilon>0$, we can find $\delta=\delta(\epsilon)>0$ such that

$$
G(z, x) \leqslant \frac{1}{p}(\eta(z)+\epsilon)|x|^{p} \text { for almost all } z \in \Omega, \text { and for all }|x| \leqslant \delta .
$$

Then for $u \in C^{1}(\bar{\Omega})$ with $\|u\|_{C^{1}(\bar{\Omega})} \leqslant \delta$, we have

$$
\begin{aligned}
\varphi(u) & =\frac{1}{p} \vartheta(u)-\frac{\hat{\lambda}_{1}}{p}\|u\|_{p}^{p}-\int_{\Omega} G(z, u) d z \\
& \geqslant \frac{1}{p}\left[\vartheta(u)-\int_{\Omega}\left[\hat{\lambda}_{1}+\eta(z)\right]|u|^{p} d z-\epsilon\|u\|^{p}\right] \quad(\text { see }(96)) \\
& \geqslant\left(c_{8}-\epsilon\right)\|u\|^{p} \text { for some } c_{8}>0 \quad \text { (see Lemma 14). }
\end{aligned}
$$

Choosing $\epsilon \in\left(0, c_{8}\right)$, we see from (97) that

$$
\begin{aligned}
& u=0 \text { is a local } C^{1}(\bar{\Omega}) \text {-minimizer of } \varphi \\
& \left.\Rightarrow u=0 \text { is a local } W^{1, p}(\Omega) \text {-minimizer of } \varphi \text { (see Proposition } 5\right) \\
& \Rightarrow C_{k}(\varphi, 0)=\delta_{k, 0} \mathbf{Z} \text { for all } k \in \mathbf{N}_{0} .
\end{aligned}
$$

Comparing (95) and (98), we infer that

$$
\begin{aligned}
& \hat{y} \neq 0, \\
& \Rightarrow \hat{y} \in C^{1}(\bar{\Omega}) \text { is the third nontrivial smooth solution of (66) }
\end{aligned}
$$

Remark 6. It would be interesting to know if, at least in the semilinear case $p=2$, one can improve the above theorem and produce a fourth nontrivial solution. The failure of the compactness condition at certain levels (see Proposition 18) does not allow us to compute the critical groups at infinity (see Bartsch and Li [3]) and therefore we cannot use the Morse relation (see (5)). So, a different approach is needed.

Acknowledgements. The authors wish to thank three anonymous referees for their corrections and remarks, which have improved the paper. This work was partially supported by the Slovenian Research Agency grants P1-0292, J1-8131, J1-7025, N10064. V. D. Rădulescu was supported by a grant of the Romanian National Authority for Scientific Research and Innovation, CNCS-UEFISCDI, project number PN-IIIP4-ID-PCE-2016-0130.

\section{References}

[1] Aizicovici, S., N. S. Papageorgiou, and V. Staicu: Degree theory of operators of monotone type and nonlinear elliptic equations with inequality constraints. - Mem. Amer. Math. Soc. 196:915, 2008.

[2] Bartolo, P., V. Benci, and D. Fortunato: Abstract critical point theorems and applications to some nonlinear problems with "strong" resonance at infinity. - Nonlinear Anal. 7, 1983, 981-1012.

[3] BARTsCh, T., and S. Li: Critical point theory for asymptotically quadratic functionals and applications to problems with resonance. - Nonlinear Anal. 28, 1997, 419-441. 
[4] Cingolani, S., and M. Degiovanni: Nontrivial solutions for $p$-Laplace equations with righthand side having $p$-linear growth at infinity. - Comm. Partial Differential Equations 30, 2005, 1191-1203.

[5] Chang, K. C.: Methods in nonlinear analysis. - Springer-Verlag, Berlin, 2005.

[6] Cuesta, M., D. De Figueiredo, and J. P. Gossez: The beginning of the Fucik spectrum for the $p$-Laplacian. - J. Differential Equations 159, 1999, 212-238.

[7] Dugundji, J.: Topology. - Allyn and Bacon Inc, Boston, 1966.

[8] Fadell, F. R., and P. Rabinowitz: Generalized cohomological index theories for Lie group actions with application to bifurcation questions for Hamiltonians systems. - Invent. Math. 45, 1978, 139-174.

[9] Gasinski, L., and N.S. Papageorgiou: Nonlinear analysis. - Chapman \& Hall/CRC, Boca Raton, Fl, 2006.

[10] Goncalves, J. V., and O. Miyagaki: Multiple nontrivial solutions of semilinear strongly resonant elliptic equations. - Nonlinear Anal. 19, 1992, 43-52.

[11] Granas, A., and J. Dugundji: Fixed point theory. - Springer-Verlag, New York, 2003.

[12] Landesman, E. M., and A. Lazer: Nonlinear perturbation of linear elliptic boundary value problems at resonance. - J. Math. Mech. 19, 1969/1970, 609-623.

[13] Liang, Z., and J. Su: Multiple solutions for semilinear elliptic boundary value problems with double resonance. - J. Math. Anal. Appl. 354, 2009, 147-158.

[14] Lieberman, G.: Boundary regularity for solutions of degenerate elliptic equations. - Nonlinear Anal. 12, 1988, 1203-1219.

[15] Motreanu, D., V. Motreanu, and N. S. Papageorgiou: Topological and variational methods with applications to nonlinear boundary value problems. - Springer, New York, 2014.

[16] Mugnai, D., and N.S. Papageorgiou: Resonant Neumann problems with indefinite weight. - Ann. Sc. Norm. Super. Pisa Cl. Sci. (5) 11, 2012, 729-788.

[17] Papageorgiou, N.S., and V.D. Rădulescu: Positive solutions for nonlinear nonhomogeneous Neumann equations of superdiffusive type. - J. Fixed Point Theory 15, 2014, 519-535.

[18] Papageorgiou, N.S., and V.D. RĂdulescu: Multiple solutions with precise sign for nonlinear parametric Robin problems. - J. Differential Equations 256, 2014, 2449-2479.

[19] Papageorgiou, N.S., and V. D. Rădulescu: Coercive and noncoercive nonlinear Neumann problems with indefinite potential. - Forum Math. 28, 2016, 545-571.

[20] Papageorgiou, N.S., and V. D. Rădulescu: Nonlinear nonhomogeneous Robin problems with superlinear reaction term. - Adv. Nonlinear Stud. 16, 2016, 737-764.

[21] Papageorgiou, N. S., and V. D. Rădulescu: Robin problems with indefinite and unbounded potential and reaction of arbirtrary growth. - Rev. Mat. Complut. 29, 2016, 91-126.

[22] Thews, K.: Nontrivial solutions of elliptic equations at resonance. - Proc. Roy. Soc. Edinburgh Sect. A 85, 1980, 119-129.

[23] Wang, X.: Neumann problems of semilinear elliptic equations involving critical Sobolev exponents. - J. Differential Equations 93, 1991, 283-310.

[24] WARD, J.: Applications of critical point theory to weakly nonlinear boundary value problems at resonance. - Houston J. Math. 10, 1984, 291-305.

Received 5 April 2017 • Accepted 28 September 2017 